\title{
Article \\ A Study on Learning Parameters in Application of Radial Basis Function Neural Network Model to Rotor Blade Design Approximation
}

\author{
Chang-Yong Song (D)
}

Citation: Song, C.-Y. A Study on Learning Parameters in Application of Radial Basis Function Neural Network Model to Rotor Blade Design Approximation. Appl. Sci. 2021, 11, 6133. https://doi.org/ 10.3390/app11136133

Academic Editors: Amerigo Capria and Theodore E. Matikas

Received: 2 April 2021

Accepted: 21 June 2021

Published: 1 July 2021

Publisher's Note: MDPI stays neutral with regard to jurisdictional claims in published maps and institutional affiliations.

Copyright: (C) 2021 by the author. Licensee MDPI, Basel, Switzerland. This article is an open access article distributed under the terms and conditions of the Creative Commons Attribution (CC BY) license (https:// creativecommons.org/licenses/by/ $4.0 /)$.
Department of Naval Architecture \& Ocean Engineering, Mokpo National University, Jeonnam 58554, Korea; cysong@mokpo.ac.kr; Tel.: +82-61-450-2732

\begin{abstract}
Meta-model sre generally applied to approximate multi-objective optimization, reliability analysis, reliability based design optimization, etc., not only in order to improve the efficiencies of numerical calculation and convergence, but also to facilitate the analysis of design sensitivity. The radial basis function neural network (RBFNN) is the meta-model employing hidden layer of radial units and output layer of linear units, and characterized by relatively fast training, generalization and compact type of networks. It is important to minimize some scattered noisy data to approximate the design space to prevent local minima in the gradient based optimization or the reliability analysis using the RBFNN. Since the noisy data must be smoothed out in order for the RBFNN to be applied as the meta-model to any actual structural design problem, the smoothing parameter must be properly determined. This study aims to identify the effect of various learning parameters including the spline smoothing parameter on the RBFNN performance regarding the design approximation. An actual rotor blade design problem was considered to investigate the characteristics of RBFNN approximation with respect to the range of spline smoothing parameter, the number of training data, and the number of hidden layers. In the RBFNN approximation of the rotor blade design, design sensitivity characteristics such as main effects were also evaluated including the performance analysis according to the variation of learning parameters. From the evaluation results of learning parameters in the rotor blade design, it was found that the number of training data had larger influence on the RBFNN meta-model accuracy than the spline smoothing parameter while the number of hidden layers had little effect on the performances of RBFNN meta-model.
\end{abstract}

Keywords: radial basis function neural network; learning parameter; design sensitivity; design approximation; rotor blade design

\section{Introduction}

The radial basis function neural network (RBFNN) is one of artificial neural network (ANN) methods employing a hidden layer of radial units and an output layer of linear units, and characterized by relatively fast training, generalization and compact type of network [1]. RBFNN has been widely used in surface and/or image constructions that typically consist of bulky size of data in the order of thousands or even millions of points. It also has been known to be suitable for highly nonlinear function approximate problems. The RBFNN has been recognized as useful method in various fields such as pattern recognition, automatic control, signal processing, approximate optimization and so on [2-5]. Meta-models are generally applied to approximate multi-objective optimization, reliability analysis, reliability-based design optimization, etc., not only in order to improve the efficiencies of numerical calculation and convergence, but also to facilitate the analysis of design sensitivity. The RBFNN has been usefully applied as the meta-model in design approximation. A sequential approximate robust design optimization with the RBFNN was proposed to find a robust optimal solution with a small number of function evaluations, not identifying a set of Pareto-optimal solution using multi-objective evolutionary 
algorithms [6]. To further reduce the computational expense of metamodel-based design optimization, a novel sequential RBF-based optimization method using virtual sample generation was proposed employing the virtual sample generation mechanism to improve the optimization efficiency [7]. However, the role of learning parameters in the RBFNN meta-model was hardly investigated in the design approximation and its application to the optimization or the reliability analysis. The reason for that the role of learning parameters in the RBFNN meta-model was not investigated closely in design approximation is that the review of parameters affecting the accuracy of the meta-model was overlooked due to the extensive numerical calculation process of optimization or reliability analysis. In Section 2.1, the author reviewed several references in which the RBFNN meta-model was utilized for the design optimizations and the reliability analyses, and it was found that the role of learning parameters in the RBFNN meta-model was hardly investigated in the design approximation. In general, the values of parameters in RBFNN meta-model have been applied empirically or adjusted through simple estimation of the accuracy of RBFNN meta-model or comparison with the results of other meta-models.

This study aims to identify the effect of various learning parameters such as the spline smoothing parameter (SSP), the number of training data, and the number of hidden layers on the performances of RBFNN meta-model regarding the design approximation of rotor blade. In particular, it is important to minimize the scattered noisy data to approximate a design space to prevent local minimum in gradient based optimization or reliability analysis using the RBFNN meta-model. Since the noisy data must be smoothed out in order for the RBFNN to be applied as the meta-model to an actual structural design problem, the SSP must be properly determined. In case of applying the RBFNN to approximate the design space, how to determine the learning parameters such as the SSP, training data, and hidden layers is important in the structural design application to enhance the performances of the meta-model. An actual rotor blade design problem was considered to investigate the characteristics of RBFNN approximation with respect to the range of SSP, the number of training data, and the number of hidden layers. In the rotor blade design approximation with the RBFNN, design sensitivity characteristics such as main effects were also evaluated including the performance analysis according to the variation of learning parameters. The present study briefly reviewed the RBFNN and its smoothing parameter theories, and then carried out the rotor blade design approximation using the RBFNN. The rotor blade design approximation included fitting of meta-models, the design sensitivity analysis, the performance analyses. The overall performances of RBFNN meta-model were evaluated using two standard accuracy measures-root mean square error and maximum error. From the design approximation results of rotor blades, it was found that the number of training data had larger influence on the RBFNN meta-model accuracy than the SSP while the number of hidden layers had little effect on the performances of RBFNN meta-model. Such study results help to suggest a reference index that can reasonably apply the learning parameter value in case of adopting the RBFNN to the design approximation. This paper is comprised of the following: Section 2 addresses literature survey related to the RBFNN and its theoretical backgrounds; Section 3 addresses the results and discusses the characteristics of the rotor blade design approximation with the RBFNN; Section 4 presents the concluding remarks of this study.

\section{RBFNN Approximation Method}

\subsection{RBFNN Meta-Model in Design Approximation}

RBFNN is originally developed to fit irregular topographic contours of geographical data [8]. RBFNN is originally generated by the same methodology but classified by various types of the basis functions. The accuracy of RBFNN model mainly depends on the applied basis function type for a given set of training and testing data. The most commonly used basis functions are linear, cubic, thin-plate spline, Gaussian, multiquadric, and inverse multiquadric functions [9]. In the research literature on RBFNN, it is applied to approximate global surfaces smoothly interpolating a given scattered data points [10]. 
As concerns optimization, RBFNN is used to solve global optimum problems where the objective function is nonlinear and nonconvex [11]. The approximate quality of RBFNN is compared with other meta-methods such as response surface methodology (RSM), Kriging and so on $[12,13]$. RBFNN model is enhanced by adding extra terms to a regular RBFNN model to increase its flexibility, based on which an optimal model could be searched for [14]. RBFNN approximation is extended using leave-one-out cross validation algorithm that can be applied in the setting of iterated moving least squares approximation and for RBF pseudo-spectral methods for the solution of partial differential equations [15]. Structure design studies that utilize meta-models have been conducted in various industrial fields. Structural analysis was performed using finite element method to review the initial design of structural safety of passive type deck support frame which was developed for the floatover installation of offshore plant, and an approximate optimization was performed using various meta-models to obtain the most efficient optimum design results [16]. Reliability analysis was performed by applying the general Monte Carlo simulation (MCS) and the Sobol sequence method, which is one of the quasi-MCS methods, to derive an efficient reliability evaluation method to ensure the structural design safety of automatic salt collector, and then the probability of reliability based on the sampling method and the accuracy of the Kriging metamodeling were compared [17]. To verify the suitability of the sensitivity analysis results of structural members and the exploration of design of experiments (DOE)based automatic ocean salt collector improvement cases, meta-modeling using the response surface method was conducted for each DOE technique, and the meta-model accuracy generated from each DOE technique was examined [18]. A novel and cheap technique was developed for finding robust optimal solutions, called confidence-based robust multiobjective optimization, using a Pareto dominance operator to differentiate search agents of meta-heuristics based on both levels of robustness and confidence [19]. An optimum design of investigated an optimal design plan to minimize design risks by applying the constraint-feasible moving least-squares method, which is a conservative approximation model. A new RBDO process was identified to ensure the constraint feasibility in the moving least squares method, and devised constraint feasibility test to quantitatively realize the feasibility of approximate constraint functions [20]. As such, many references have reported various studies related to approximate design optimization using the RBFNN, but the role of the learning parameters including the SSP in the RBFNN application has barely been identified. Previous studies on the SSP were also mostly theoretical studies or application studies to signal processing and image approximation [15,21-23]. Based on the aeroelastic analysis, the design enhancement of wing structure was carried out by targeting an optimal wing structure able to withstand flight loads, and simultaneously with stiffness and inertia distributions leading to a configuration free from flutter within the flight envelope [24]. In the design strategy for the wing structure that aimed at finding a first optimal solution in terms of the thickness of composite components, two-level optimization was carried out [25]. This study aims to suggest the reference index that can reasonably apply the learning parameters such as the range of spline smoothing parameter, the number of training data, and the number of hidden layers in case of adopting the RBFNN to the design approximation problems.

\subsection{Theoretical Background of RBFNN and SPP}

A brain is composed of networks of neurons. A typical neuron receives an input, which is either excitation or inhabitation, from many other neurons. As its net excitation reaches a certain level, the neuron fires. The firing is propagated through a branching axon to many other neurons, where it in turn acts as input to those neurons. The neuron's firing is thought of its output as a binary or continuous valued quantity. The neural network models are algorithms for cognitive tasks, such as learning and optimization, which are based on concepts derived from research in the nature of the brain. In the neural network model, the neurons are generally organized into layers. The inputs to the neural network model are presented to the input layer, and the outputs are generated as signals of the 
output layer. Since the numeric activations and signals comprise vectors, the input vector applied to the input layer generates an output signals vector across the output layer. These signals pass through one or more intermediate or hidden layers which transform the signals depending upon the neuron signal functions [26-28].

In this study, RBFNN was used to generate a meta-model that can be applied to approximate design optimization, and the performances of the generated meta-model were evaluated according to the variation of the learning parameters such as the SSP value, the number of training data, and the number of hidden layers. RBFNN has been developed for scattered multivariate data interpolation $[1,8]$. This technique uses linear combinations of a radial symmetric function based on Euclidean distance to approximate response functions. Let $x_{1}, \cdots, x_{n} \in \Omega \subset R^{n}$ be a given set of nodes, and a set of any RBF basis function, $g(x)$, is to be:

$$
g_{j}(x)=\phi\left(\left\|x-x_{j}\right\|\right) \in R, j=1, \cdots, n
$$

In Equation (1), $\phi$ is power spline basis function and the norm $\left\|x-x_{j}\right\|$ is Euclidean distance. By using of Euclidean distance, the data of input layer are classified as homogeneous cluster. Power spline basis function is expressed as follows:

$$
\phi=r^{c_{j}}
$$

where $c$ is a shape function variable with positive constant value.

Given interpolation data $y_{1}, \cdots, y_{n} \in R$ at data points $x_{1}, \cdots, x_{n} \in \Omega \subset R^{n}, R B F N N$ interpolant $f^{\prime}(x)$ through the network learning process as shown in Figure 1 is defined as follows:

$$
f^{\prime}(x)=\sum_{j=1}^{n} \alpha_{j} g_{j}(x)+\alpha_{n+1}
$$

This study adopted one layer, two layers, and three layers respectively in the neural network architecture in order to evaluate the effect of the number of hidden layers on the performance of RBFNN for the rotor blade design approximation. The interpolant is obtained by solving the system of $n+1$ linear equation as follows:

$$
\sum_{j=1}^{n} \alpha_{j} g_{j}\left(x_{i}\right)+\alpha_{n+1}=y_{i}, i=1, \cdots, n
$$

for $n+1$ unknown expansion coefficients $\alpha_{j}$.

The SSP is a parameter value used to relax the over-fitting feature that happens in a meta-model approximated via the RBFNN in case of passing through every single data point over whole design space. Its primary purpose is to smooth out noisy data. By not going through every data point, meta-model can be effectively fitted as a smoothing function and employed in an approximate optimization that may be easier to solve. The value specified by the SSP averages the output values of data points that are clustered in the normalized filter domain. The SSP trades smoothness of the fitted function against fidelity to the data and can be expressed by the following equation:

$$
\min \rho\|s\|^{2}+\frac{1}{n} \sum_{i=1}^{n}\left(s\left(x_{i}\right)-f^{\prime}\left(x_{i}\right)\right)^{2}
$$

where $\rho$ is positive smoothing control parameter and the norm $\|s\|$ is smoothness penalty. From Equation (5), larger value of $\rho$ increases the amount of smoothness relative to the average deviation from the data squared. The value of SSP is estimated generally through iterative evaluation to minimize the maximum deviation while satisfying the accuracy of a given RBFNN meta-model in the approximate design optimization. 


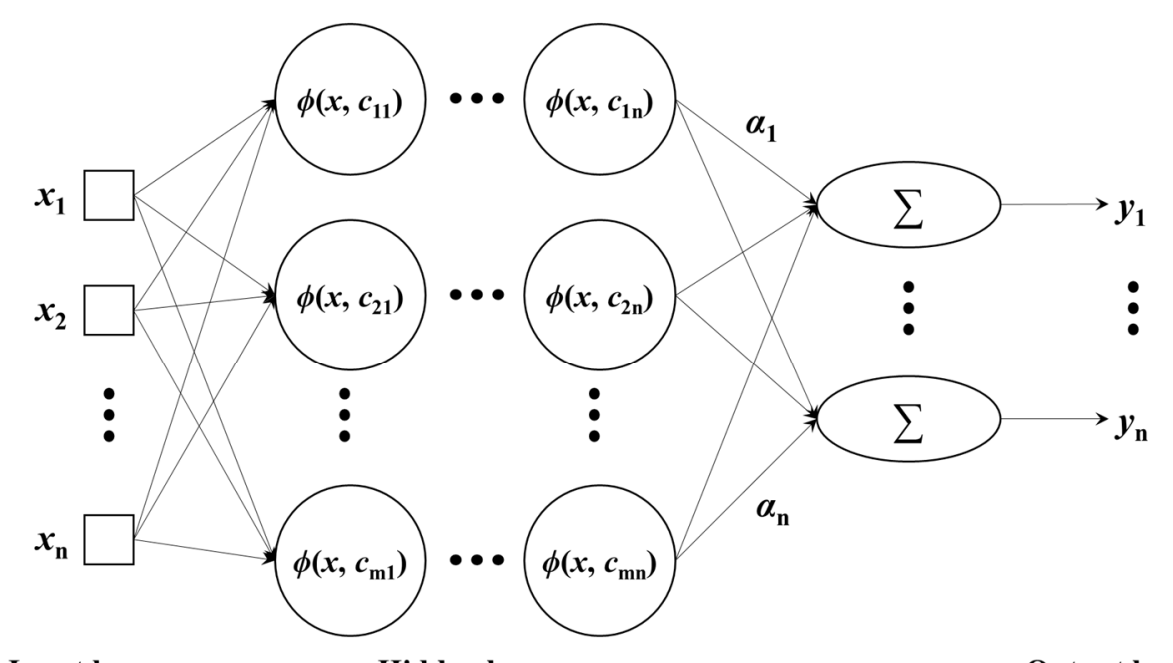

Input layer

Hidden layers

Output layer

Figure 1. Neural network learning process of RBFNN.

The overall performance was evaluated using two standard accuracy measures-root mean square error (RMSE) and maximum error (MAX). RMSE provides an overall error measure over the entire solution space and is defined as follows:

$$
\mathrm{RMSE}=\sqrt{\frac{1}{n} \sum_{i=1}^{n}\left(f\left(x_{i}\right)-f^{\prime}\left(x_{i}\right)\right)^{2}}
$$

The MAX is indicative of local deviations and also expressed as follows:

$$
\operatorname{MAX}=\max _{i}\left|f\left(x_{i}\right)-f^{\prime}\left(x_{i}\right)\right|
$$

where $f\left(x_{i}\right)$ denotes the exact function value for the test point $x_{i}, f^{\prime}\left(x_{i}\right)$ is the corresponding predicted value using the meta-model, and $n$ is the number of test points chosen for calculating the error measure [29,30]. Ideally, it would be favorable that both of error measures are to be as small as possible for higher accuracy. The RMSE is the accuracy measure commonly used in case of dealing with the difference between the predicted value of the meta-model and the actual response value and is suitable for expressing the overall accuracy of the meta-model. The RMSE is used for the loss function in the training process. On the other hand, because the MAX calculates the maximum error among the predicted values of the meta-model, local accuracy can be confirmed. Since the RBFNN can be applied not only to linear design space but also to approximation of highly nonlinear design space, both the overall accuracy and the local accuracy should be reviewed simultaneously. Therefore, it is reasonable to evaluate the accuracy of the RBFNN meta-model by applying both the RMSE and the MAX. For the rotor blade design problem, statistical analysis was also performed to evaluate the main effects of design variables on the output responses of design performance functions. Since RBFNN is the interpolant meta-model, results such as RSME and MAX are feature values for the metamodel accuracy, and the feature values depend on of the learning parameters such as the SSP value, the number of training data, and the number of hidden layers. Therefore, in case that new variables are to be applied in the design process, the learning parameters should be redefined. Hyperparameters used for the RBFNN modeling were summarized in Table 1. 
Table 1. Hyperparameter values.

\begin{tabular}{cc}
\hline Hyperparameter & Value \\
\hline Learning rate & 0.7 \\
Number of training iteration & 10 \\
Number of hidden layers & 1 to 3 \\
Weight initialization & $-1 / 25,1 / 25$ \\
Spline smoothing parameter & 0.0 to 0.1 \\
\hline
\end{tabular}

In Table 1, the learning rate determines how fast it moves in the direction of the gradient. The number of training iterations was used to determine the early stopping of learning and applied to prevent overfitting.

\subsection{Mathematical Example}

Although it would be a special case that a high nonlinear problem such as mathematical example exists in an actual structural design problem, Ackley's path function was considered as the mathematical example for the accuracy evaluation of RBFNN meta-model regarding to the SSP. Consider the following Ackley's path function with two variables [31]:

$$
\begin{gathered}
f(x)=-20 \exp \left(-0.2 \sqrt{\frac{x_{1}^{2}+x_{2}^{2}}{2}}\right)-\exp \left\{\frac{\cos \left[2 \pi\left(x_{1}\right)\right]+\cos \left[2 \pi\left(x_{2}\right)\right]}{2}\right\}+20+e \\
-2.0 \leq x_{1}, x_{2} \leq 2.0
\end{gathered}
$$

where $f(x)$ is the mathematical function that presents a high nonlinearity with multimodal, and $x_{1}$ and $x_{2}$ are design variables. Both 200 training data and 30 testing data were randomly generated within the range of the design variables, for this function, and then the RBFNN based meta-models were fitted on 2-D and 3-D design spaces as shown in Figures 2 and 3.

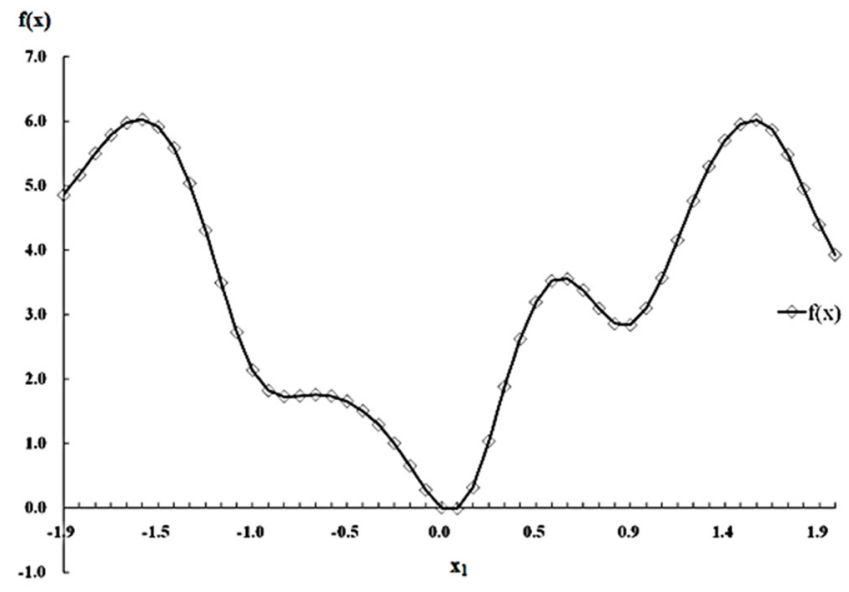

(a) $x_{1}-f(x)$

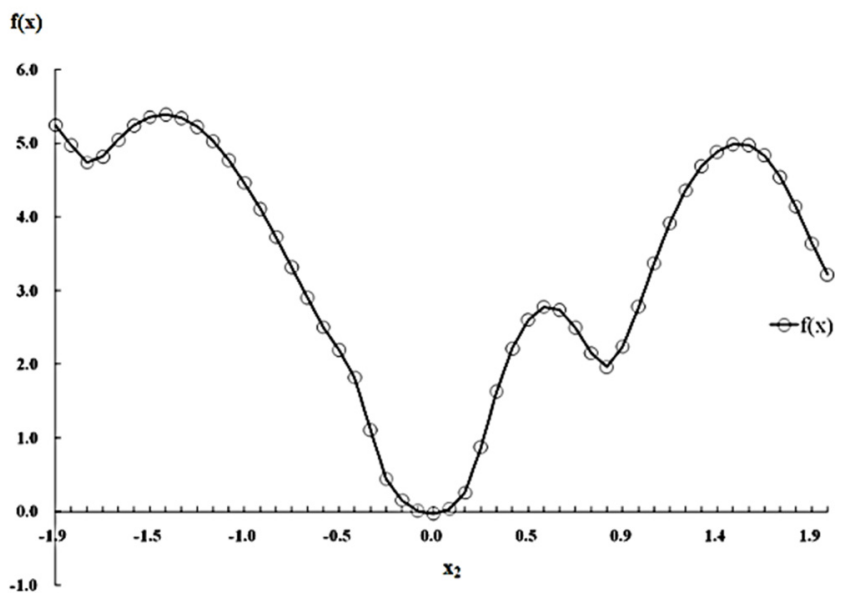

(b) $x_{2}-f(x)$

Figure 2. Meta-models on 2-D design space for Ackley's path function. 


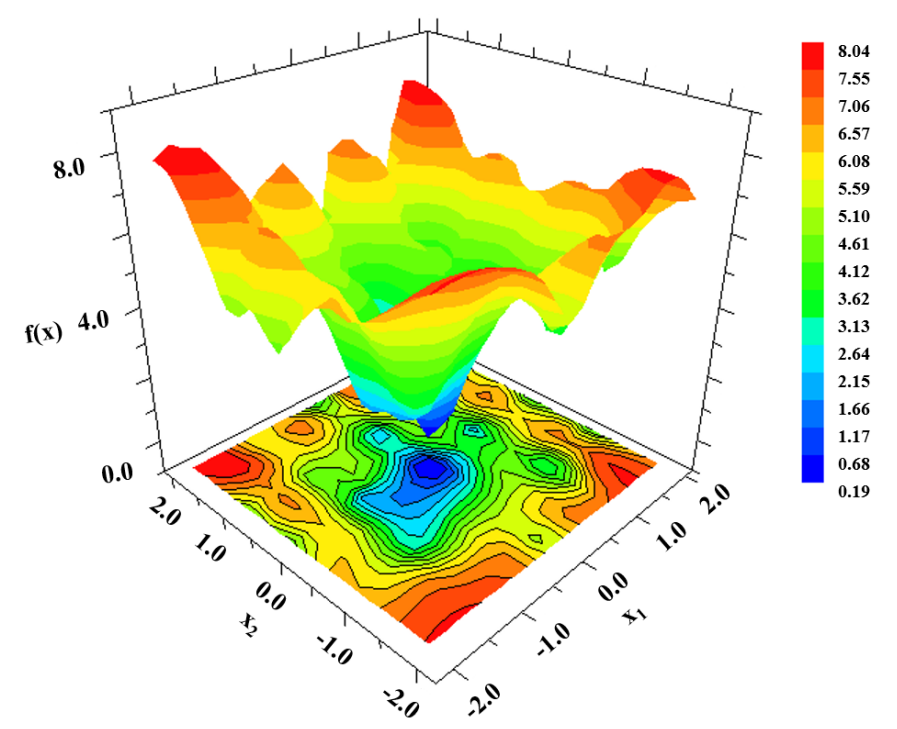

Figure 3. Meta-model on 3-D design space for Ackley's path function.

From Figures 2 and 3, it was clearly understood that the mathematical example has highly nonlinearity, and the RBFNN was apt for approximating such multimodal problem with large size training and testing data. As concerns the SSP, error analyses were carried out to evaluate the variation of performance according to the SSP values. The SSP values were varied from zero to 0.1 with 0.025 step size, and the accuracy measures of RBFNN meta-model such as RMSE and MAX were calculated using Equations (6) and (7). Their results were presented in Table 1 and Figure 4.

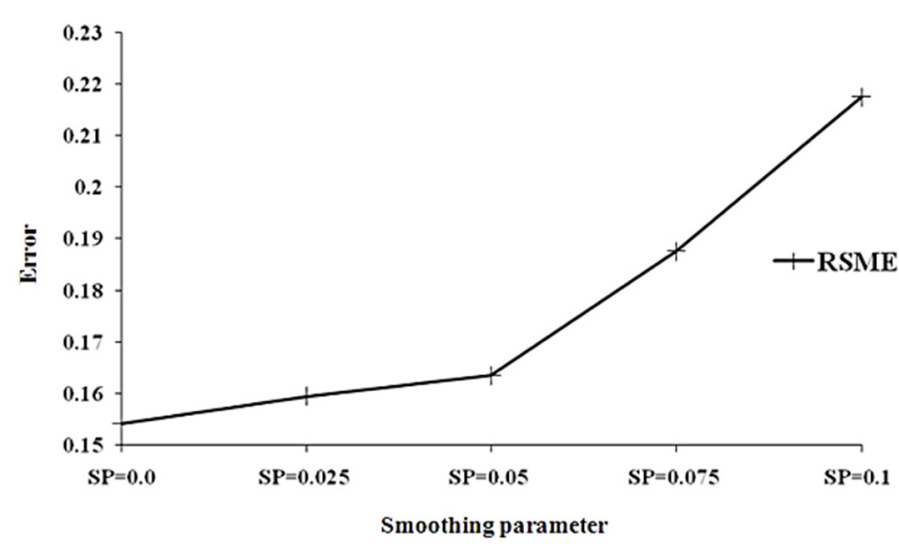

(a) RMSE

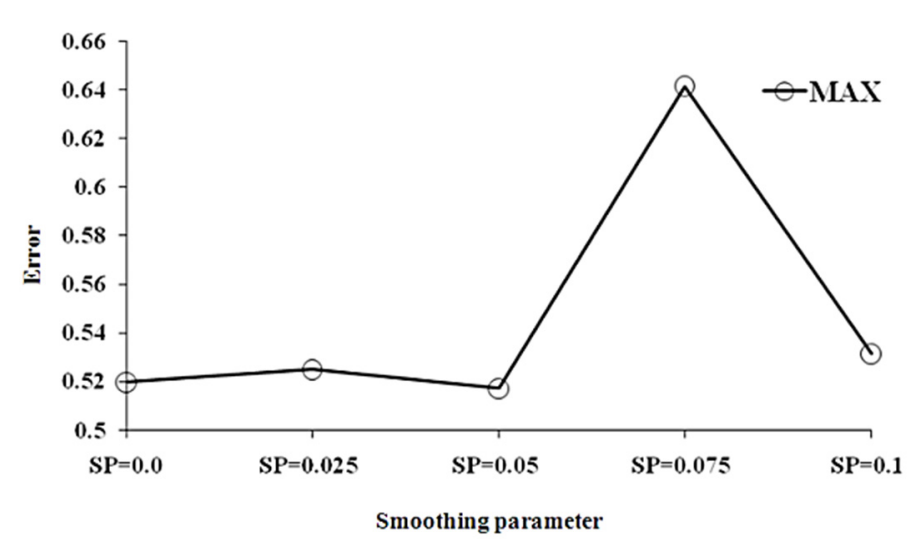

(b) Maximum error

Figure 4. Performance comparison according to variation of SSP in Ackley's path function.

As shown in Table 2 and Figure 4, the RMSE results were distributed from 0.154 to 0.218 according to the variation of SSP. The RMSE results were varied slightly until the SSP value was increased up to 0.05 , whereas the RMSE values were increased significantly as the SSP value exceeded 0.05 . The RMSE results also showed the inferior overall accuracy according to the increment of SSP. When approximating a design space with very high nonlinearity such as the mathematical example, very careful selection is necessary to apply the SSP value in order to guarantee the accuracy of RBFNN meta-model. As shown in Figure $4 \mathrm{~b}$, Local deviation was slightly changed except 0.075 of SSP value. The MAX value at 0.075 of SSP value was increased by $24 \%$ comparing to the result at 0.0 of SSP value. The abrupt increment of the MAX at 0.075 of SSP value was due to the highly nonlinearity of the Ackley's path function. 
Table 2. Results of accuracy according to variation of SSP in Ackley's path function.

\begin{tabular}{ccc}
\hline SSP Value & RMSE & MAX \\
\hline 0.0 & 0.1542 & 0.5198 \\
0.025 & 0.1594 & 0.5249 \\
0.050 & 0.1635 & 0.5172 \\
0.075 & 0.1876 & 0.6416 \\
0.1 & 0.2176 & 0.5316 \\
\hline
\end{tabular}

The characteristics of error scattering for the RBFNN meta-model were also evaluated according to the SSP values. The results of error scattering analyses were shown in Figure 5.

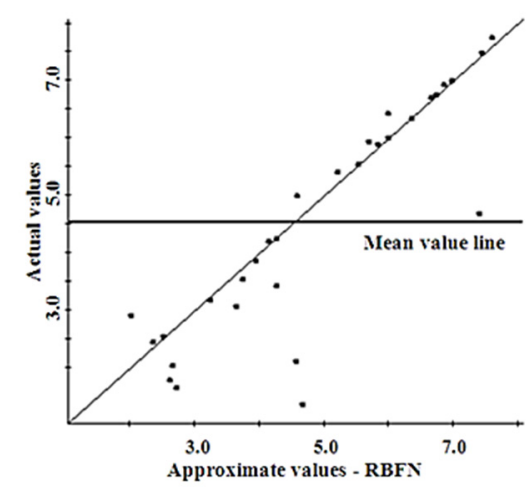

(a) Smoothing parameter $=0.0$

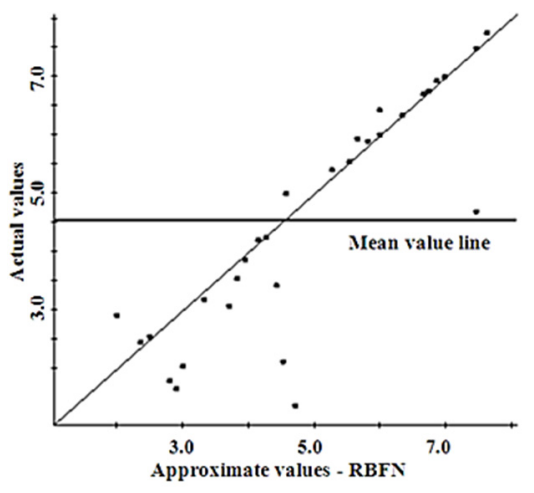

(b) Smoothing parameter $=0.025$

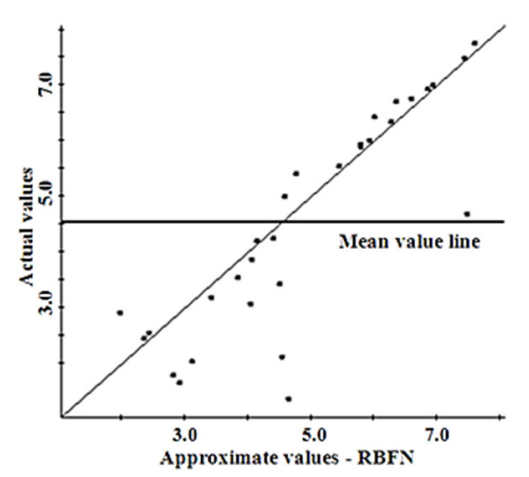

(c) Smoothing parameter $=0.05$

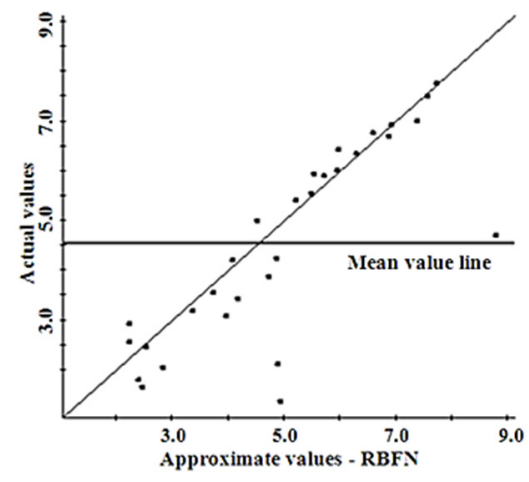

(d) Smoothing parameter $=0.075$

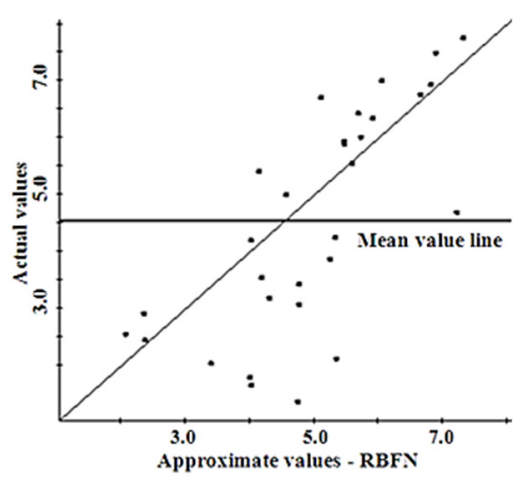

(e) Smoothing parameter $=0.1$

Figure 5. Error scattering analysis according to variation of SSP in Ackley's path function.

Similar to the RMSE results, the error scattering results were varied slightly until the SSP value was increased up to 0.05 , whereas the error scattering points deviated from the actual values were increased significantly as the SSP value exceeded 0.05. As shown in Figure $5 \mathrm{~d}, \mathrm{e}$, the error scattering points were deviated widely according to the increment of SSP. 


\section{Rotor Blade Design Approximation with RBFNN}

In the rotor blade design, it is important to minimize the distance between aerodynamic and shear centers in order to reduce both the flutter instability and the restrictions on the flight envelope. Also, the weight of rotor blade should be improved while the structural stiffness properties are almost maintained at the level of the baseline design. The design optimization problem of rotor blade can be formulated as follows:

$$
\begin{gathered}
\text { Minimize } f=|\alpha-\gamma| \\
\text { subject to } \frac{m}{m_{\text {base }}} \leq 1 \\
0.95 \leq \frac{\mathrm{GJ}}{\mathrm{GJ}_{\text {base }}} \leq 1.05 \\
0.95 \leq \frac{\mathrm{EI}_{22}}{\left(\mathrm{EI}_{22}\right)_{\text {base }}} \leq 1.05 \\
0.95 \leq \frac{\mathrm{EI}_{33}}{\left(\mathrm{EI}_{33}\right)_{\text {base }}} \leq 1.05
\end{gathered}
$$

where $\alpha$ is the aerodynamic center, and $\gamma$ is the shear center. The properties GJ, $\mathrm{EI}_{22}$ and $\mathrm{EI}_{33}$ are torsional, flap-wise bending, and chord-wise bending stiffnesses, respectively. The notation $m$ is mass per unit length. The subscript base denotes the value representing the baseline model. An application of optimization methods to the structural design problems of rotor blade often requires a large amount of numerical costs. An alternative for reducing the numerical cost in the process of design optimization is to employ the meta-model by establishing the accurate levels of approximate objective and constraint functions over the exploratory design space. Using rationally constructed meta-models, near-optimal approximate solutions can be obtained in addition to the estimation of design behaviors, that is, the sensitivity-like information.

The practical design problem of rotor blade was chosen to investigate the characteristics of the RBFNN approximation with respect to the learning parameters such as the range of spline smoothing parameter, the number of training data, and the number of hidden layers. The RBFNN based meta-models were generated for the rotor blade design problem. The architecture of RBFNN consisted of one to three layers in the hidden layers to evaluate the effect of the number of hidden layers on the performance of RBFNN. The number of neurons in that layer was defined as the mean of the neurons in the input and output layers referring to the related research book [28]. Each neural network in the RBFNN was trained by back-propagation algorithm which is one of the most reliable algorithms having such characteristics as the simplicity of implementation and the ability to quickly generate networks that have the capability to generalize [28]. Error analyses were carried out to evaluate the variation of performance according to the variation learning parameters. In order to complete the RBFNN meta-modeling for the rotor blade design problem, the calculation time was $8.3 \mathrm{~h}$ using a server equipped with six core Intel Xeon CPUs and 128 GB RAM.

In order to investigate the characteristics of the RBFNN approximation with respect to the learning parameters for the practical design problem with a number of performance functions, the design problem of composite rotor blade was explored [32]. The composite rotor blade was designed to use for the tilt rotor aircraft combines the advantages of vertical takeoff and landing capabilities. The design performance functions of rotor blade were calculated a rotor blade cross-sectional modeling and analysis program, which is Variational Asymptotic Beam Section (VABS) [33] developing for analyzing the composite blade structure using an equivalent beam model. In the VABS program, the cross-sectional stiffness matrix is calculated from a two-dimensional finite element discretization. In this study, the VABS was used for obtaining both training and testing data for use in RBFNN based meta-modeling. Regarding to the cross-section of rotor blade with specific 
geometry information, a NURBS curve was generated for a given number of points, and the non-uniform rational B-spline (NURBS) was transformed into the cross-section of rotor blades with specific geometry data. Such information was adopted to calculate the design performance functions of rotor blade by VABS. The structure design configuration and the design variables of the rotor blade were shown in Figure 6.

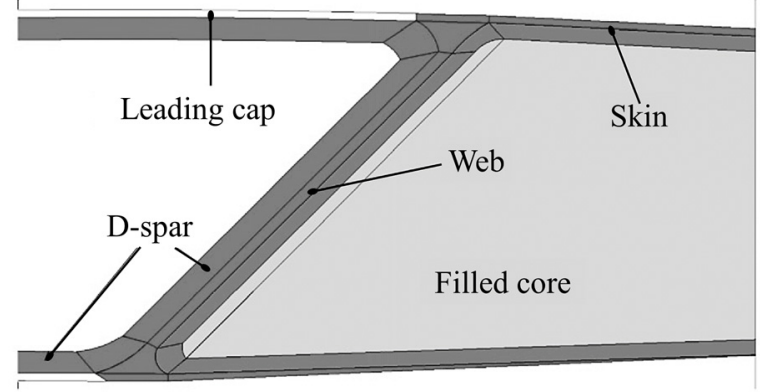

(a) Sectional configuration

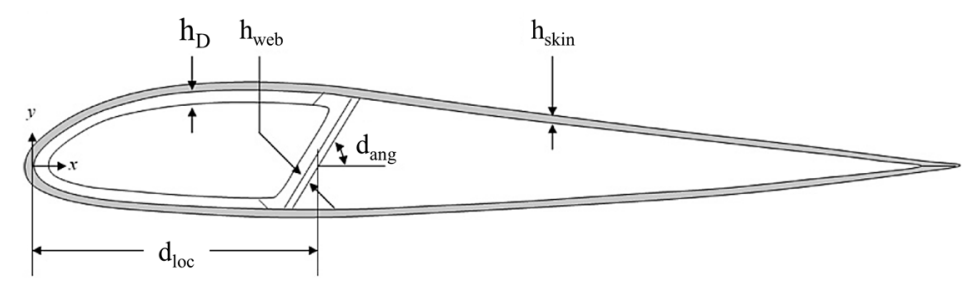

(b) Design variables

Figure 6. Structure design configuration and design variables of rotor blade.

As shown in Figure 6, the design variables were thicknesses of D-spar $\left(h_{D}\right)$, skin $\left(h_{\text {skin }}\right)$, and web $\left(\mathrm{h}_{\mathrm{web}}\right)$, as well as the web location $\left(\mathrm{d}_{\text {loc }}\right)$ and the web connection orientation $\left(\mathrm{d}_{\mathrm{ang}}\right)$. Both initial values and design range for the design variables were represented in Table 3 .

Table 3. Initial value and design range in design variable.

\begin{tabular}{cccc}
\hline Design Variable & Lower Bound & Initial Design & Upper Bound \\
\hline $\mathrm{h}_{\mathrm{D}}$ (in.) & 0.3 & 0.35 & 0.4 \\
$\mathrm{~h}_{\text {skin }}$ (in.) & 0.03 & 0.04 & 0.05 \\
$\mathrm{~h}_{\text {web }}$ (in.) & 0.01 & 0.04 & 0.05 \\
$\mathrm{~d}_{\text {loc }}$ (in.) & -9.0 & -8.4 & -7.0 \\
$\mathrm{~d}_{\text {ang }}$ (deg.) & 60 & 90 & 120 \\
\hline
\end{tabular}

In the calculation of design performances, the chord length was considered $20.2 \mathrm{in}$., and the aerodynamic center was at the quarter chord. In the structure design problem of composite rotor blade, the distance between aerodynamic and shear centers should be minimized to avoid flutter instability of the rotor blade, and to avert the restrictions on the flight envelope. As design performance functions, the structural mass of rotor blade should be minimized while some important internal structural stiffness properties such as torsional (GJ), flap-wise bending $\left(\mathrm{EI}_{22}\right)$ and chord-wise bending stiffnesses $\left(\mathrm{EI}_{33}\right)$ were almost maintained at the level of the initial design, i.e., within $\pm 5 \%$ variations.

In the present study, the RBFNN-based approximation was carried out for the design performance functions. With 500 training data and 90 testing data, the RBFNN based metamodels were fitted for each design performance function. The fitted RBFNN meta-models of rotor blade design are shown in Figures 7-11. 


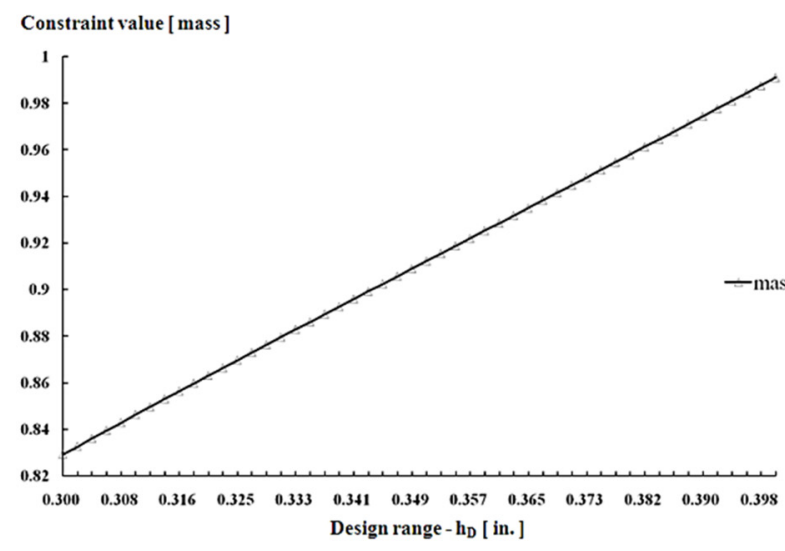

Constraint value [GJ]

Constraint value [ $\mathrm{EI}_{2:}$ ]
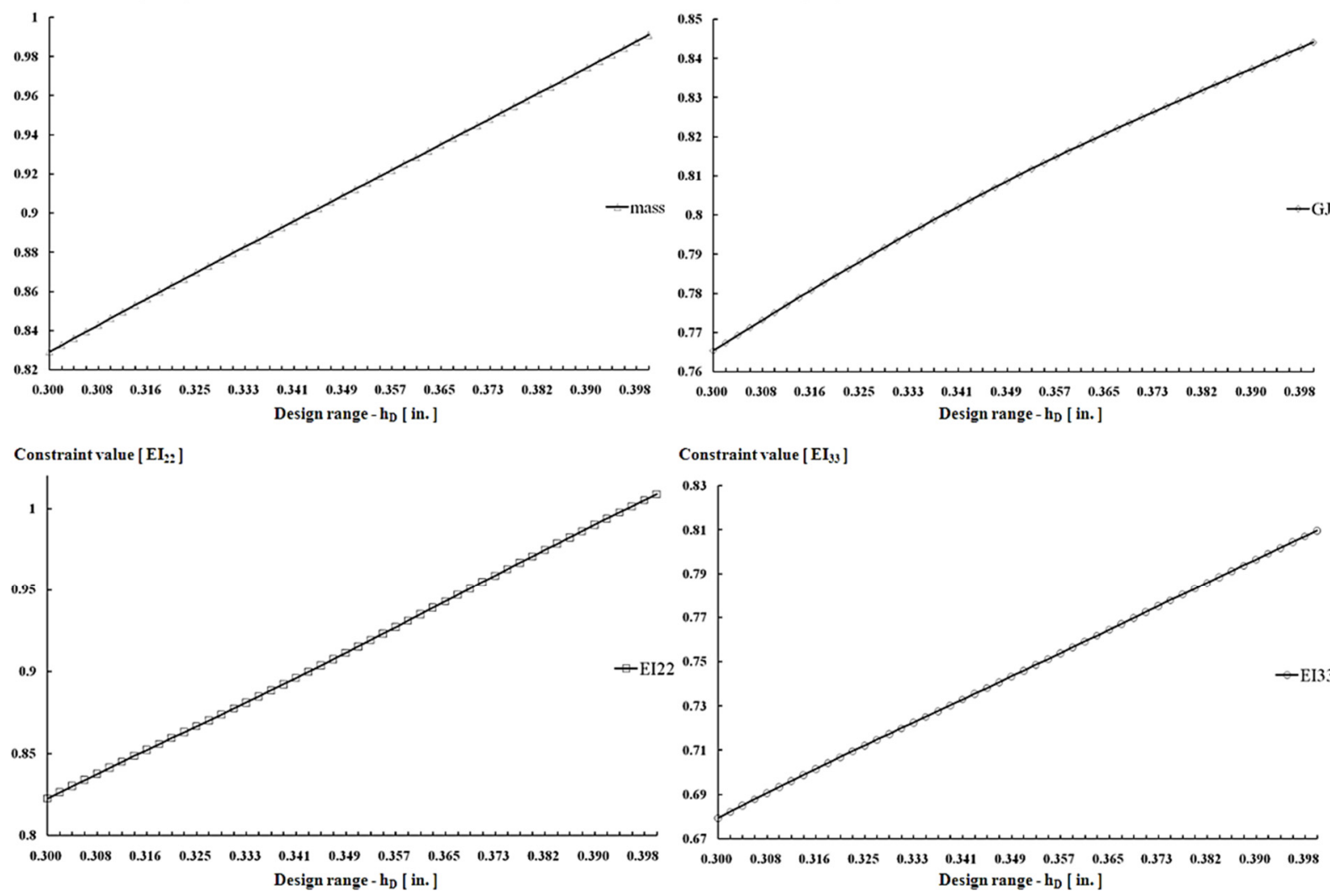

Constraint value [ $\left.\mathrm{EI}_{33}\right]$

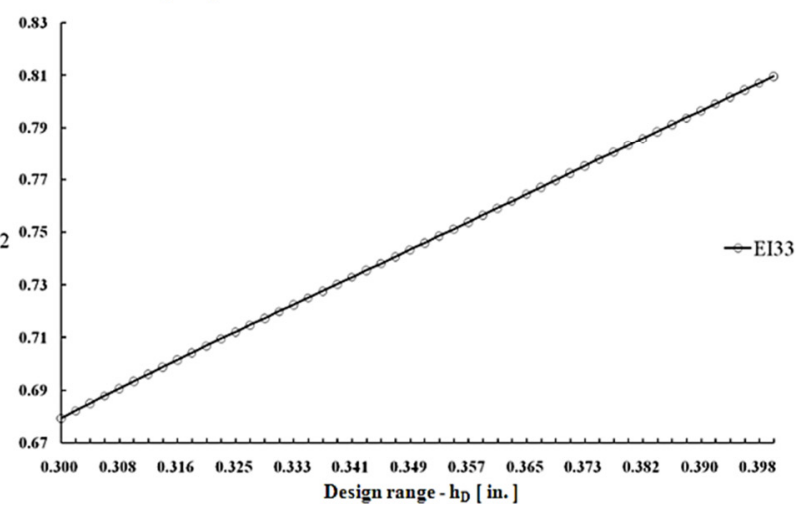

Figure 7. Meta-models for design variable- $\mathrm{h}_{\mathrm{D}}$.
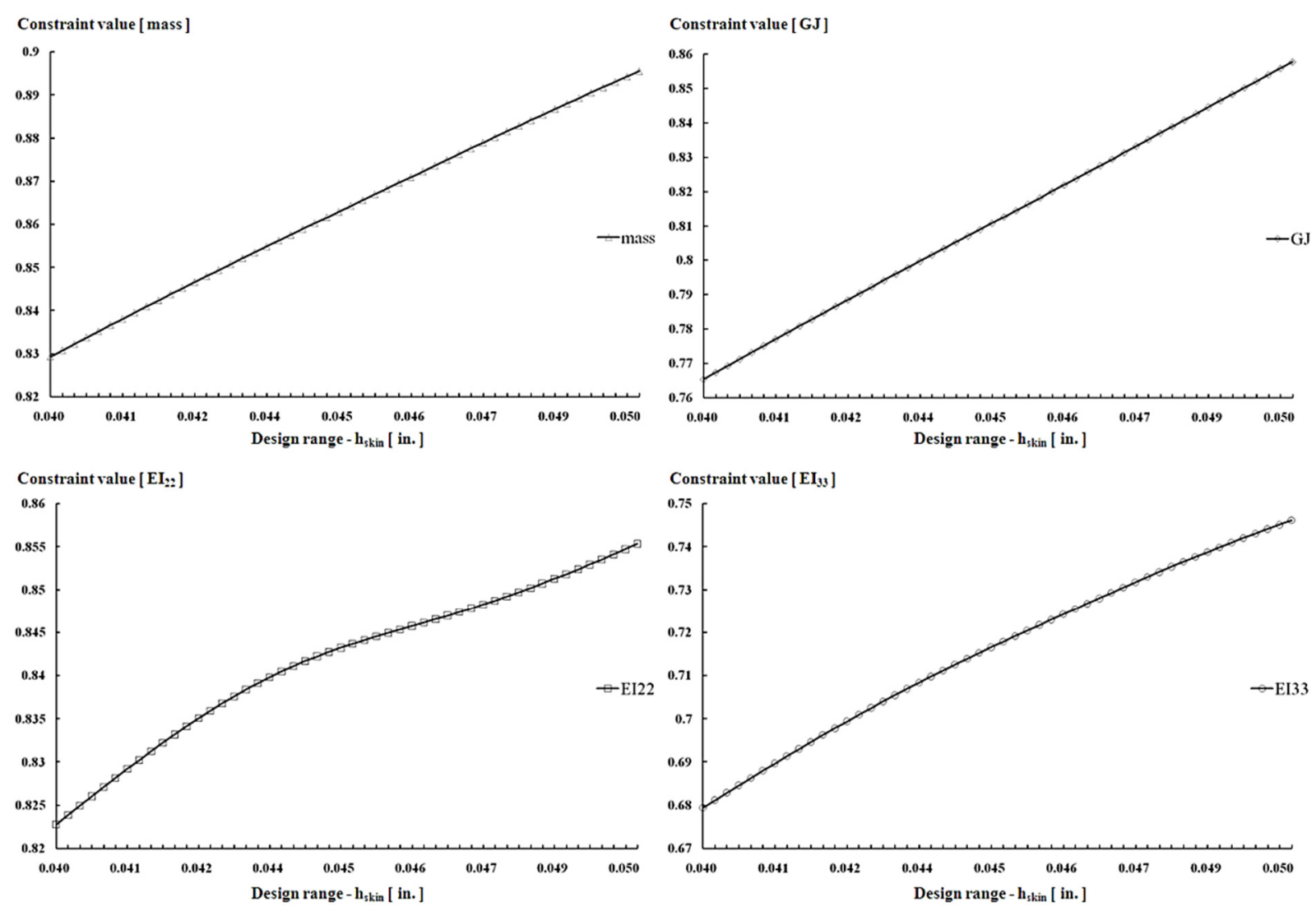

Figure 8. Meta-models for design variable $-\mathrm{h}_{\text {skin }}$. 


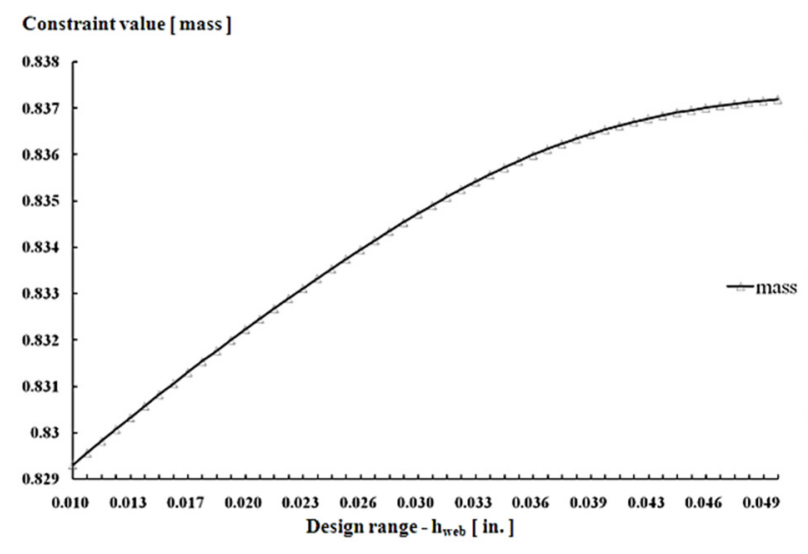

Constraint value [GJ]
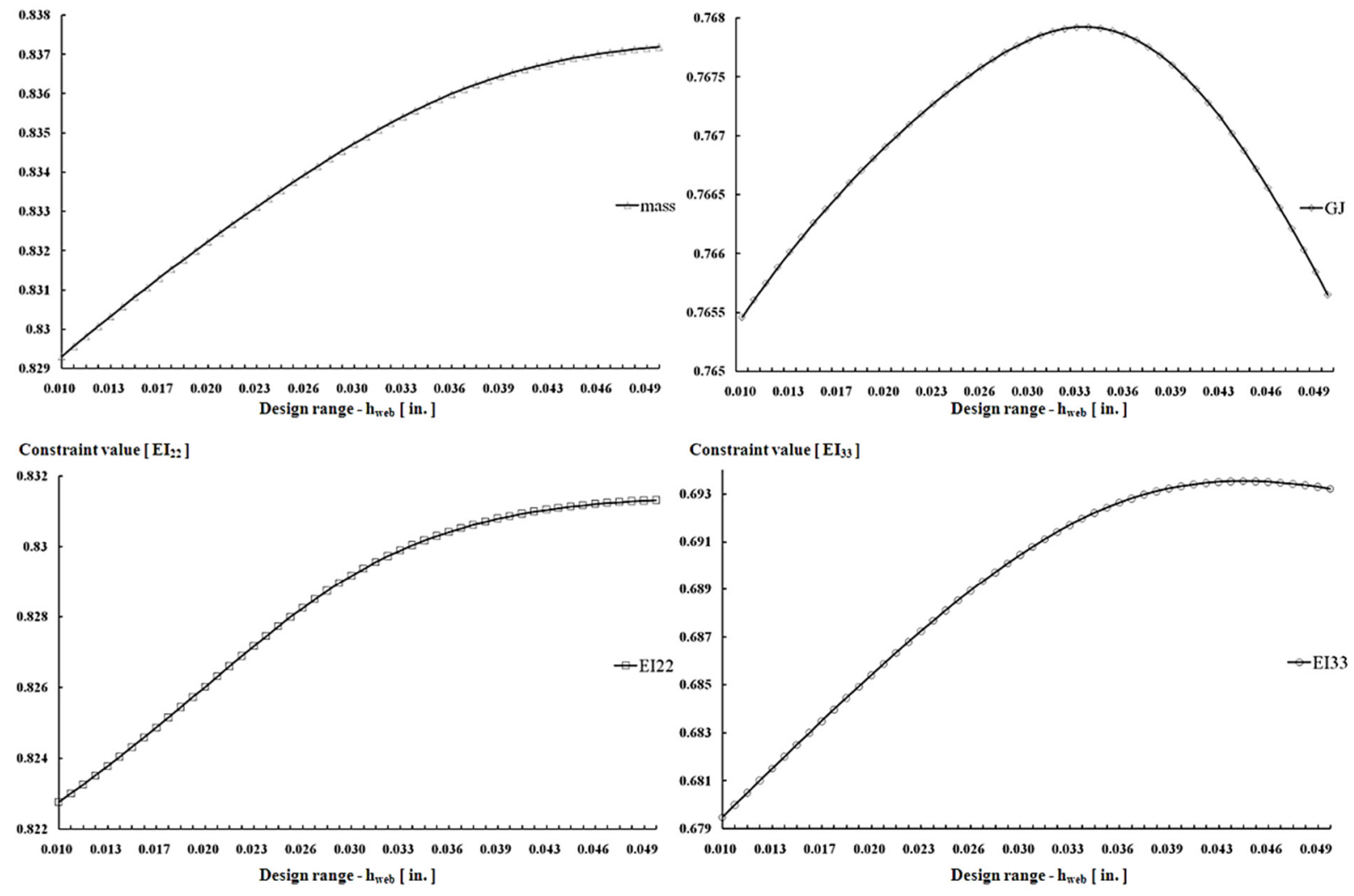

Figure 9. Meta-models for design variable $-h_{w e b}$.

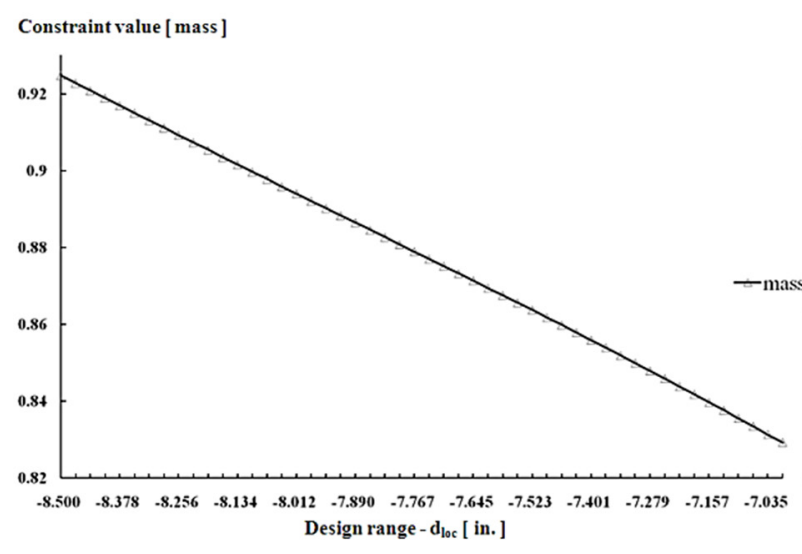

Constraint value $[\mathrm{GJ}]$

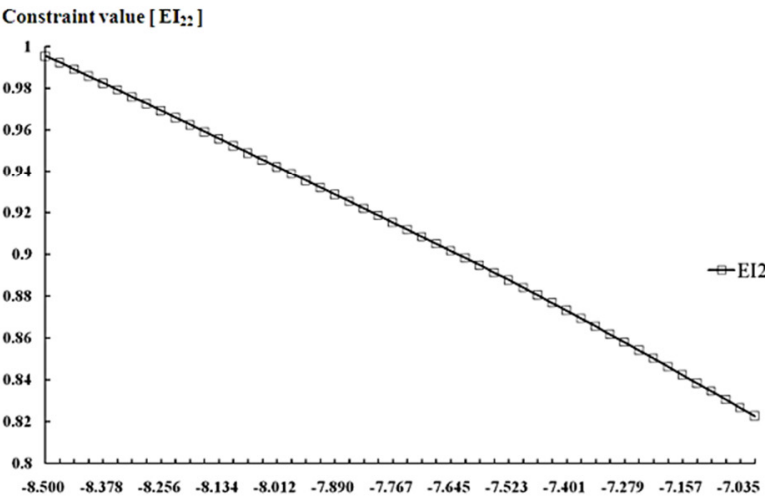
Design range - $d_{\text {loc }}[$ in. ]

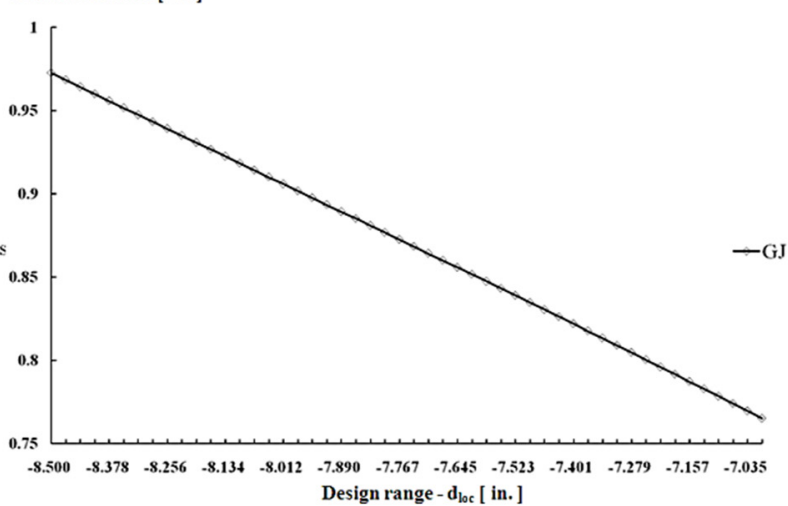

Constraint value $\left[\mathrm{EI}_{33}\right]$

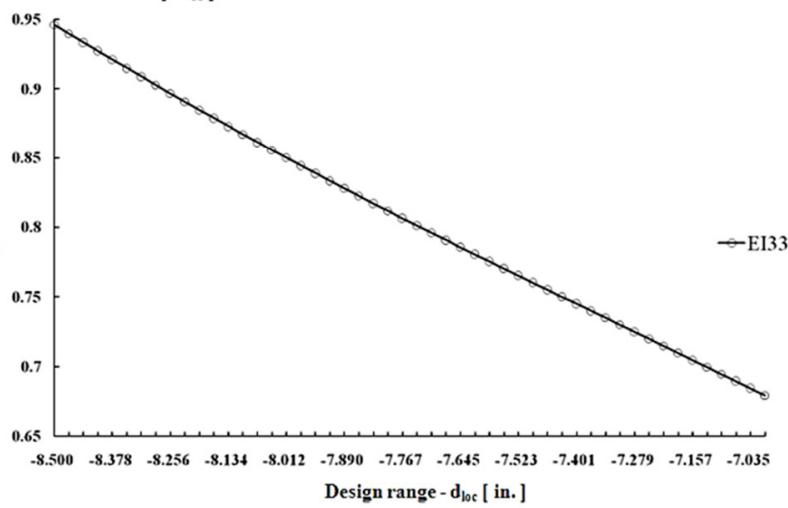

Figure 10. Meta-models for design variable $-\mathrm{d}_{\mathrm{loc}}$. 

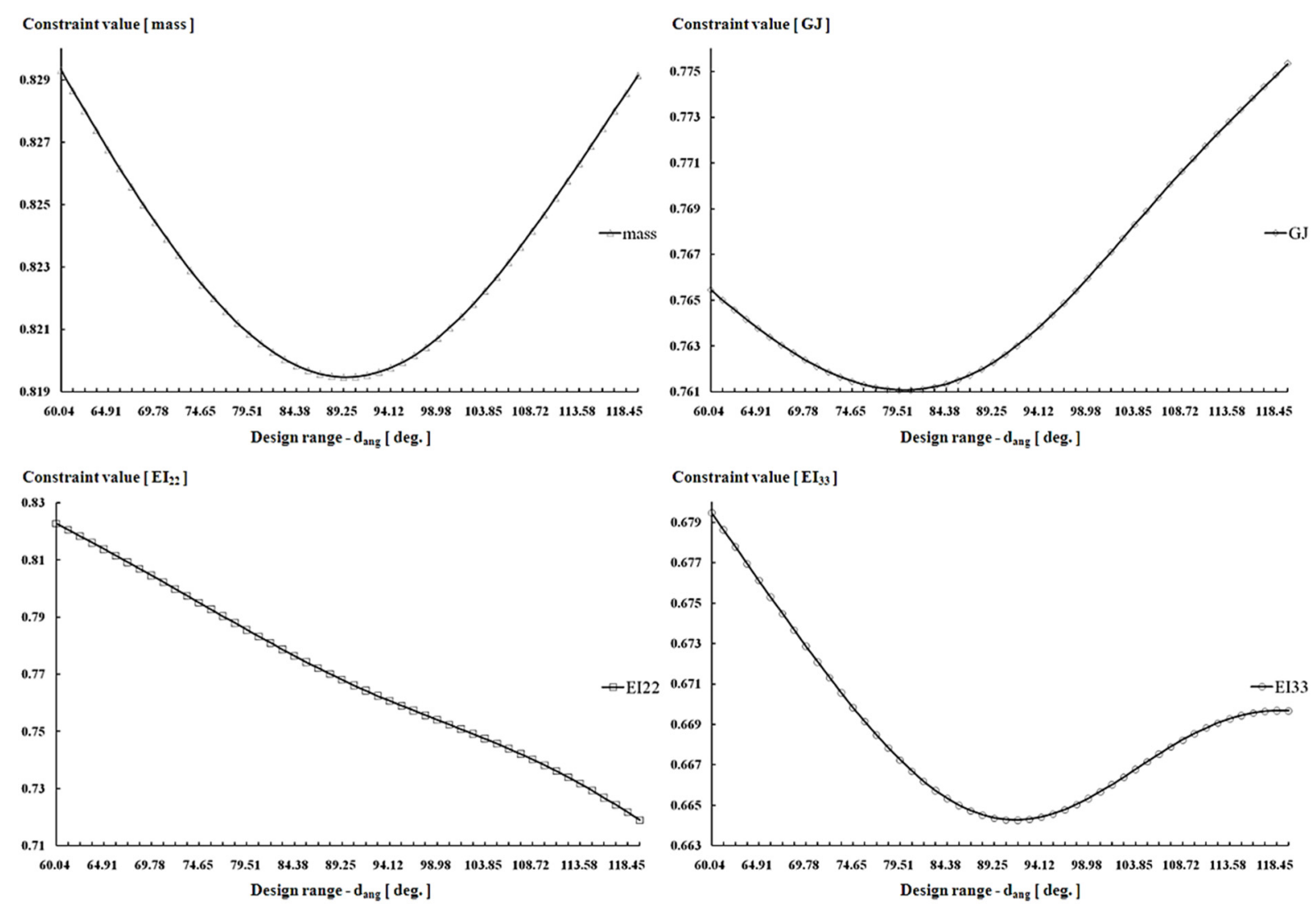

Figure 11. Meta-models for design variable $-d_{a n g}$.

From the meta-modeling results, remarkable multi-modal variations were found in the relations between two design variables $\left(h_{w e b}\right.$ and $\left.d_{a n g}\right)$ and the design performance functions. In other design variables such as the thicknesses of D-spar $\left(h_{D}\right)$, the $s k i n\left(h_{\text {skin }}\right)$ and the web location, their meta-modeling results represented almost linear characteristics. Statistical analyses were also performed to evaluate the main effects of design variables on the responses of design performance functions. The results of statistical analyses were shown in Figure 12.

The main effects of $h_{D}$ and $d_{l o c}$ among the design performance functions were higher than other design variables. The most sensitive design variable on the mass and the $\mathrm{EI}_{22}$ was the $\mathrm{h}_{\mathrm{D}}$, and the main effect values on the mass and the $\mathrm{EI}_{22}$ were $47 \%$ and $44 \%$, respectively. The most sensitive design variable on the GJ and the $\mathrm{EI}_{33}$ was the $\mathrm{d}_{\text {loc }}$, and the main effect values on the $\mathrm{GJ}$ and the $\mathrm{EI}_{33}$ were $-52 \%$ and $-48 \%$, respectively. The statistical results also showed that $d_{l o c}$ and $d_{\text {ang }}$ had negative effects on all the design performance functions while other design variables had positive effects. From the main effect results, it was found that the design performances of rotor blade were increased as the $h_{D}$ value was increased and the $\mathrm{d}_{\mathrm{loc}}$ value was decreased.

The error analyses were carried out to evaluate the variation of performance according to the variation of learning parameters such as the SSP value, the number of training data, and the number of hidden layers. The SSP values were varied from zero to 0.1 with 0.025 step size, and the accuracy measures such as RMSE and MAX were analyzed. Their results were represented in Table 4 and Figures 13 and 14.

As shown in Table 4 and Figure 13, the RMSEs of Mass, GJ, $\mathrm{EI}_{22}$, and $\mathrm{EI}_{33}$ were distributed in the range of 0.00590 to $0.00593,0.00645$ to $0.00647,0.02021$ to 0.02026 , and 0.02375 to 0.02384 , respectively, according to the variation of SSP. The MAXs of Mass, GJ, $\mathrm{EI}_{22}$, and $\mathrm{EI}_{33}$ were distributed in the range of 0.02473 to $0.02483,0.01567$ to $0.01589,0.09622$ to 0.09637, and 0.09031 to 0.09046, respectively, as represented in Table 4 and Figure 14. From the results of error analyses, it was clear that the variation of accuracy occurred in 
the slight magnitude because the rotor blade design problem had relatively a low multimodal variation as represented in the meta-modeling results and the statistical analysis. Although the influence of SSP was not significant in the RBFNN approximation of the actual rotor blade design problem, it could be seen that the accuracy of the approximation was increased based on RMSE as the SSP was applied in the range of 0.075 to 0.1. The results of error scattering were also evaluated according to the SSP values. The results of error scattering analyses were shown in Figure 15.

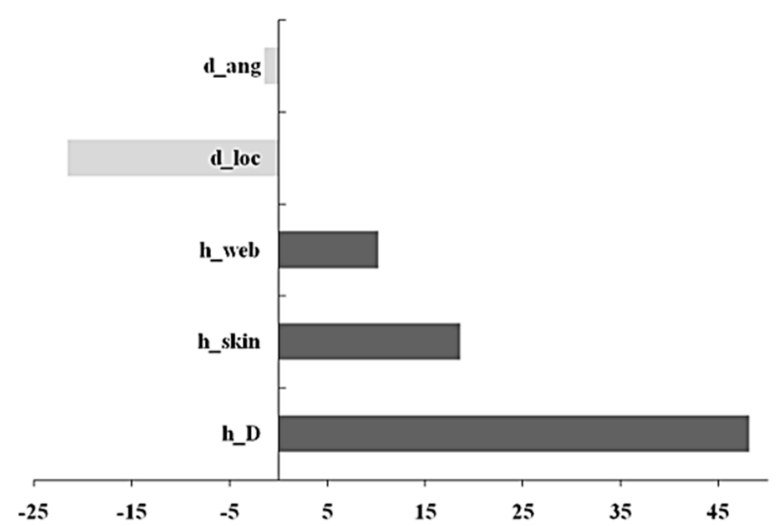

(a) Main effects on mass (\%)

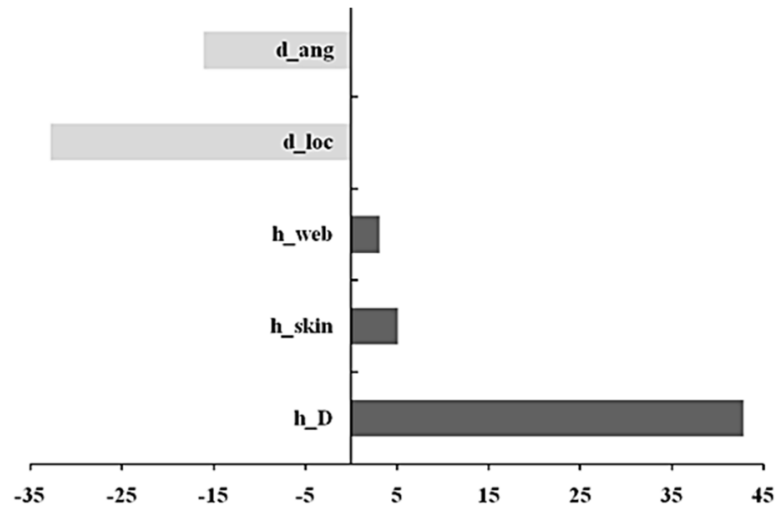

(c) Main effects on $\mathrm{EI}_{22}(\%)$

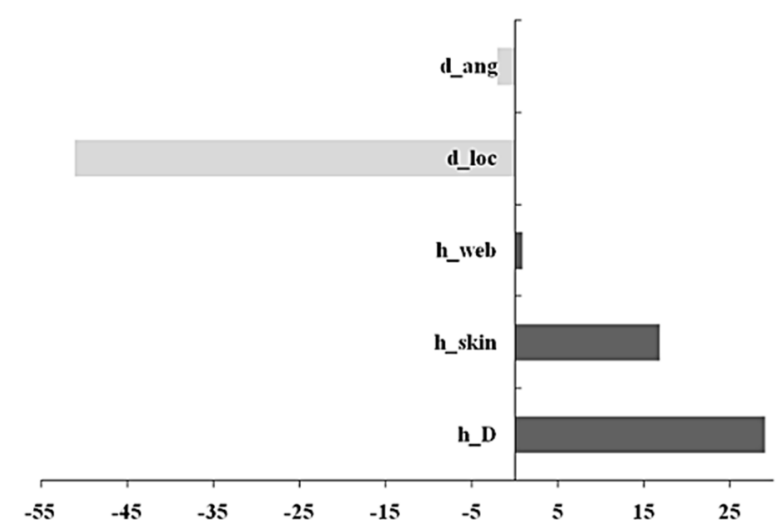

(b) Main effects on GJ (\%)

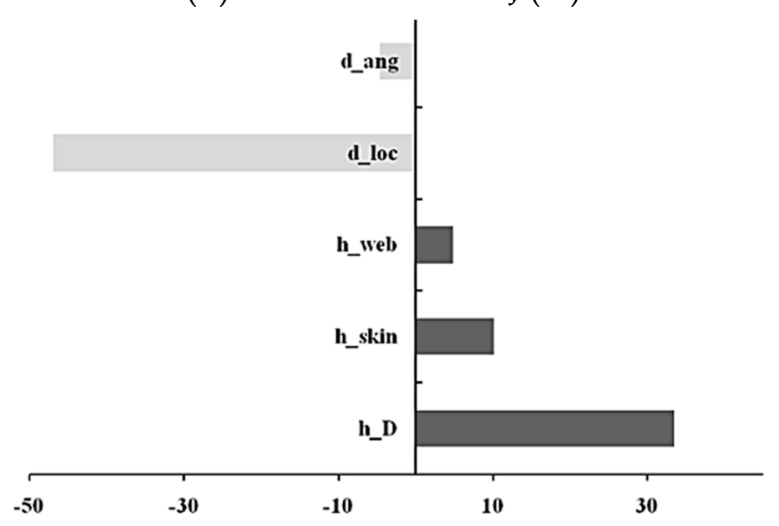

(d) Main effects on $\mathrm{EI}_{33}(\%)$

Figure 12. Main effects on design performance functions.

Table 4. Results of accuracy according to variation of SPP.

\begin{tabular}{cccccc}
\hline Error Type & SSP Value & Mass & GJ & EI $_{\mathbf{2 2}}$ & EI $_{\mathbf{3 3}}$ \\
\hline \multirow{4}{*}{ RMSE } & 0.0 & 0.00590 & 0.00646 & 0.02023 & 0.02375 \\
& 0.025 & 0.00593 & 0.00647 & 0.02026 & 0.02384 \\
& 0.050 & 0.00592 & 0.00647 & 0.02026 & 0.02383 \\
& 0.075 & 0.00592 & 0.00645 & 0.02025 & 0.02382 \\
MAX & 0.1 & 0.00592 & 0.00645 & 0.02021 & 0.02380 \\
& 0.0 & 0.02473 & 0.01567 & 0.09622 & 0.09031 \\
& 0.025 & 0.02483 & 0.01598 & 0.09641 & 0.09053 \\
& 0.050 & 0.02482 & 0.01594 & 0.09638 & 0.09050 \\
& 0.075 & 0.02481 & 0.01589 & 0.09636 & 0.09047 \\
& 0.1 & 0.02483 & 0.01589 & 0.09637 & 0.09046 \\
\hline
\end{tabular}




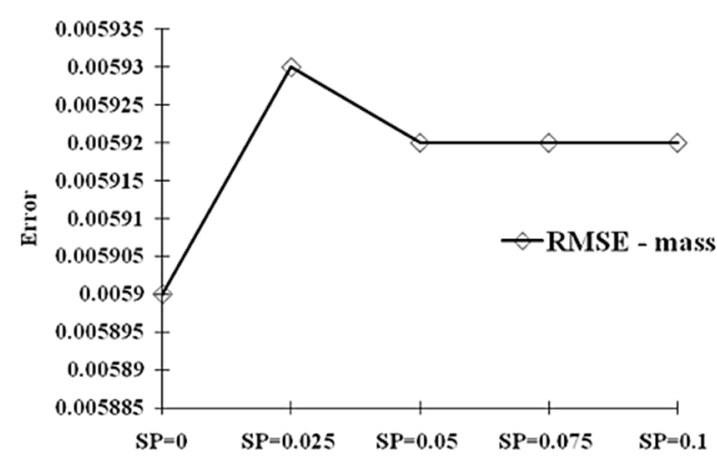

(a) RMSE on mass

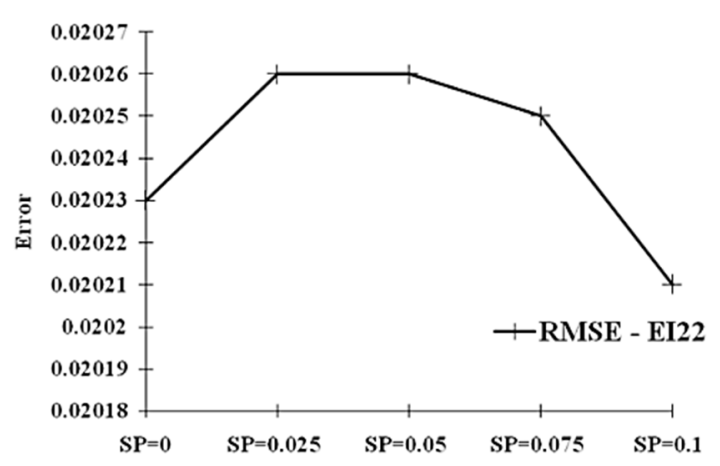

(c) RMSE on $\mathrm{EI}_{22}$

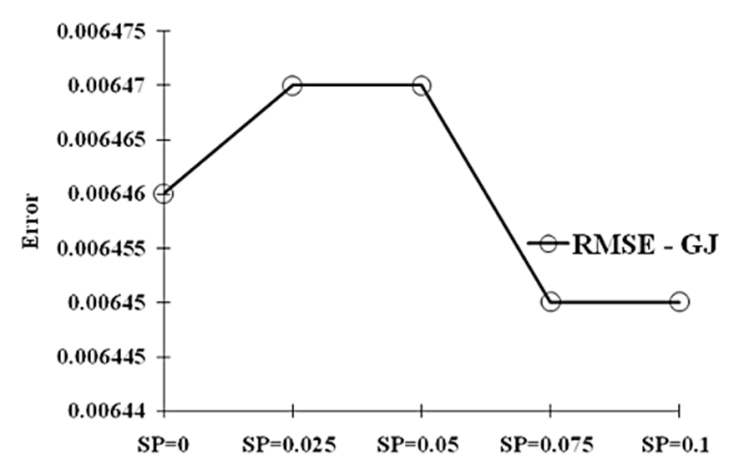

(b) RMSE on GJ

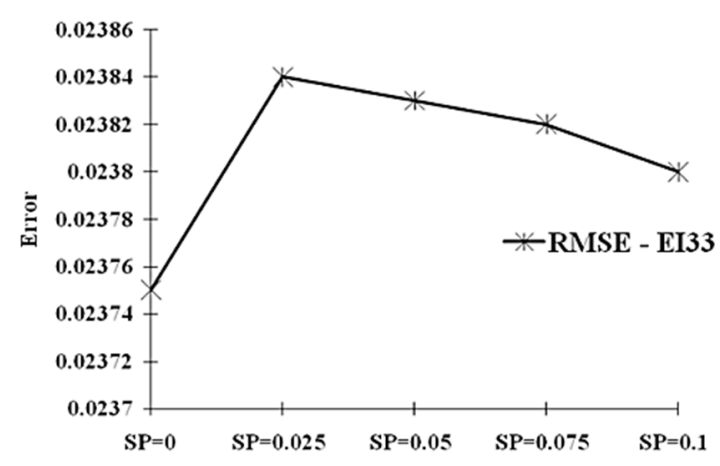

(d) RMSE on $\mathrm{EI}_{33}$

Figure 13. RMSE according to variation of SPP.

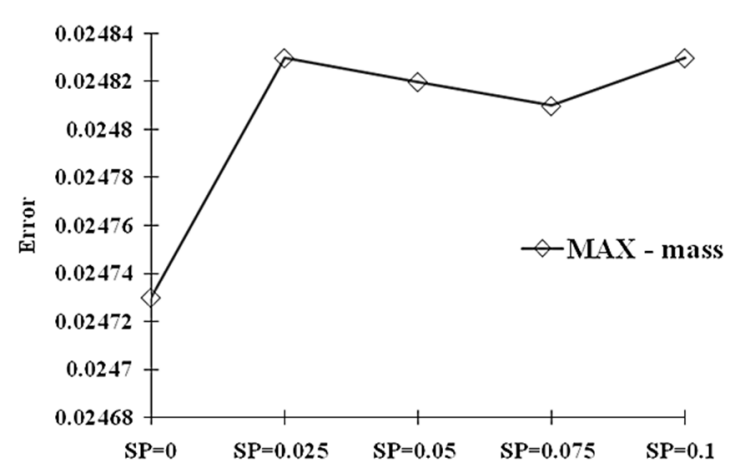

(a) MAX on mass

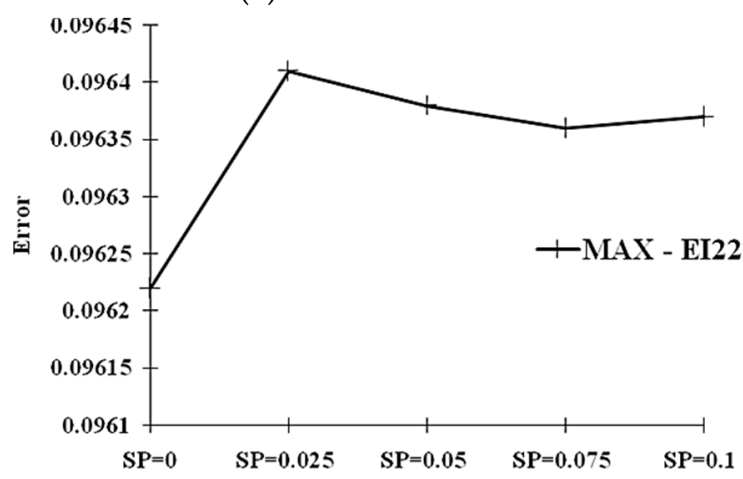

(c) MAX on $\mathrm{EI}_{22}$

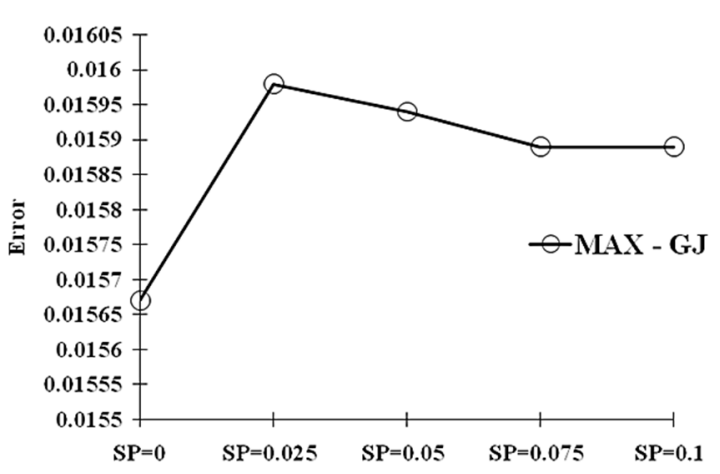

(b) MAX on GJ

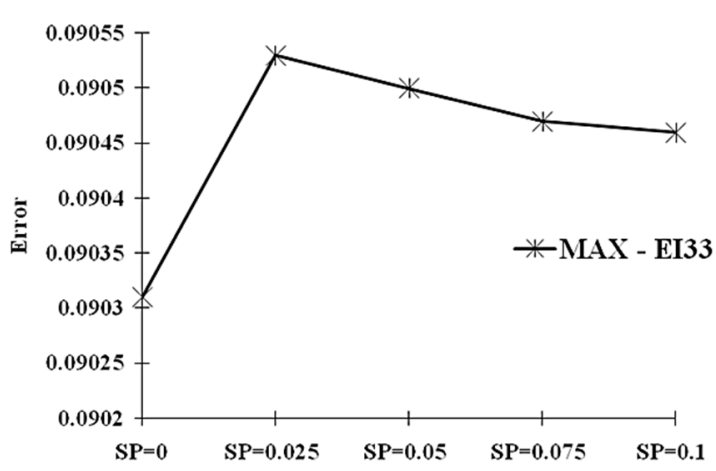

(d) $\mathrm{MAX}$ on $\mathrm{EI}_{33}$

Figure 14. Maximum error according to variation of SPP. 


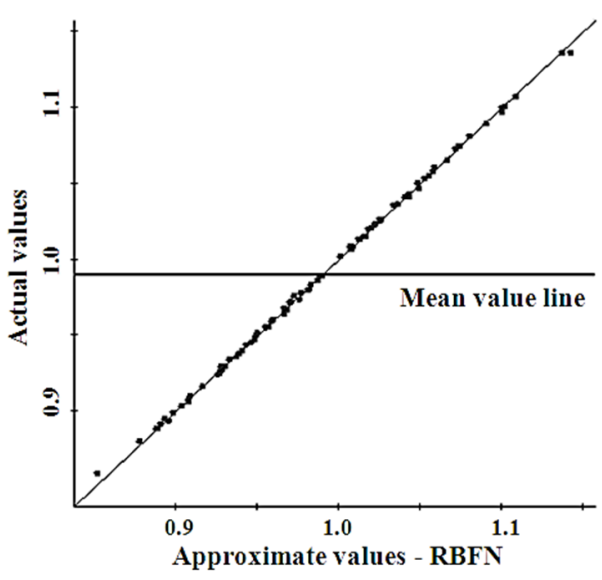

(a) mass

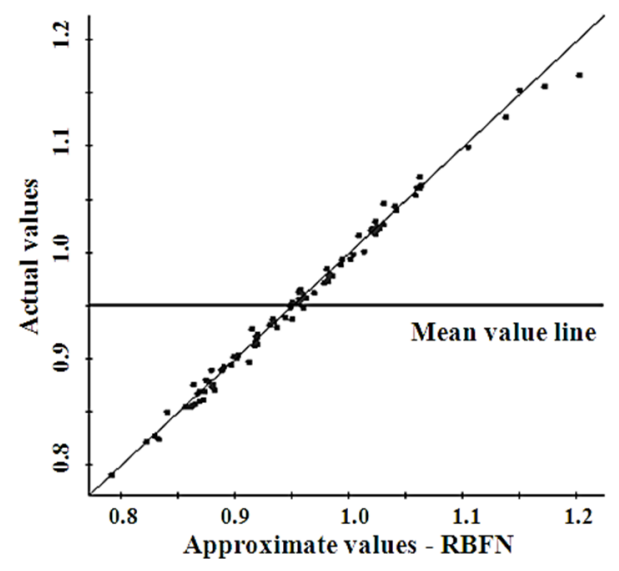

(c) $\mathrm{EI}_{22}$

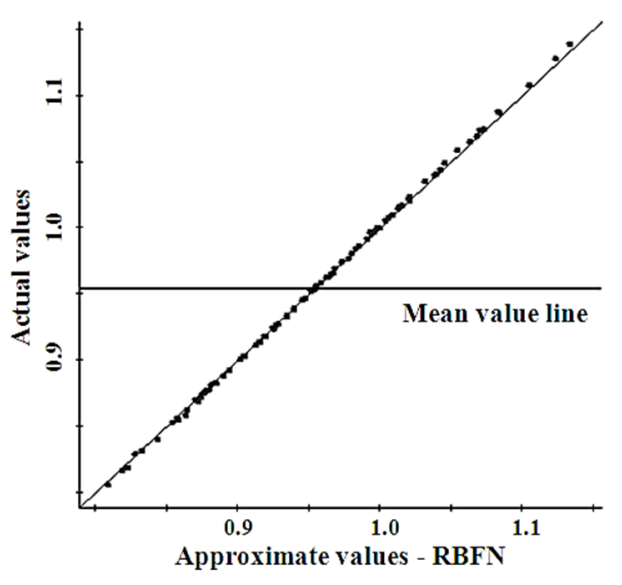

(b) GJ

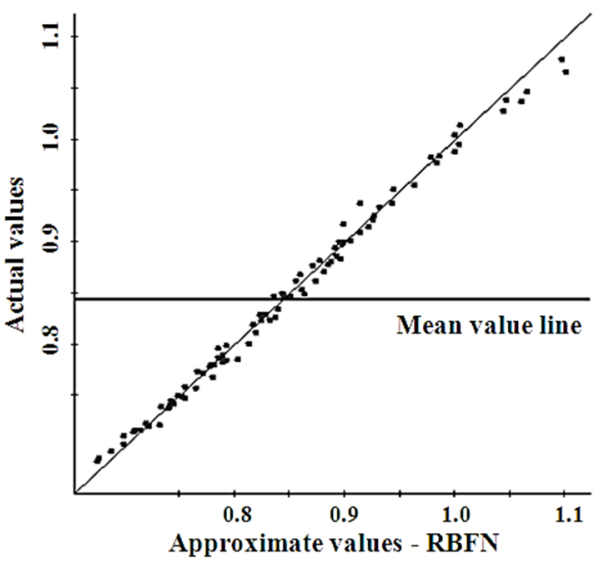

(d) $\mathrm{EI}_{33}$

Figure 15. Error scattering analysis for design performance functions.

Figure 15 showed only for the zero SSP, and it was sufficient to represent the characteristics of error scattering because the variation of accuracy is tiny in the rotor blade design problem.

In the context of the error analyses of rotor blade design problem, the performance characteristics according to the variation of the number of training data were secondly evaluated. The initial number of 500 training data was varied up to 200 training data with 100 data step size. The results of RMSE and MAX were shown in Table 5 and Figures 16 and 17.

Table 5. Results of accuracy according to variation of training data.

\begin{tabular}{cccccc}
\hline Error Type & \# of Training Data & Mass & GJ & EI $_{\mathbf{2 2}}$ & EI $_{\mathbf{3 3}}$ \\
\hline \multirow{4}{*}{ RMSE } & 500 & 0.00590 & 0.00646 & 0.02023 & 0.02375 \\
& 400 & 0.00618 & 0.00693 & 0.02178 & 0.02521 \\
& 300 & 0.00631 & 0.00733 & 0.02326 & 0.02539 \\
200 & 0.00643 & 0.00737 & 0.02334 & 0.02787 \\
\hline \multirow{3}{*}{ MAX } & 500 & 0.02473 & 0.01567 & 0.09622 & 0.09031 \\
& 400 & 0.02499 & 0.01592 & 0.10349 & 0.09434 \\
& 300 & 0.02498 & 0.01845 & 0.09126 & 0.08605 \\
& 200 & 0.02509 & 0.01979 & 0.08138 & 0.08316 \\
\hline
\end{tabular}




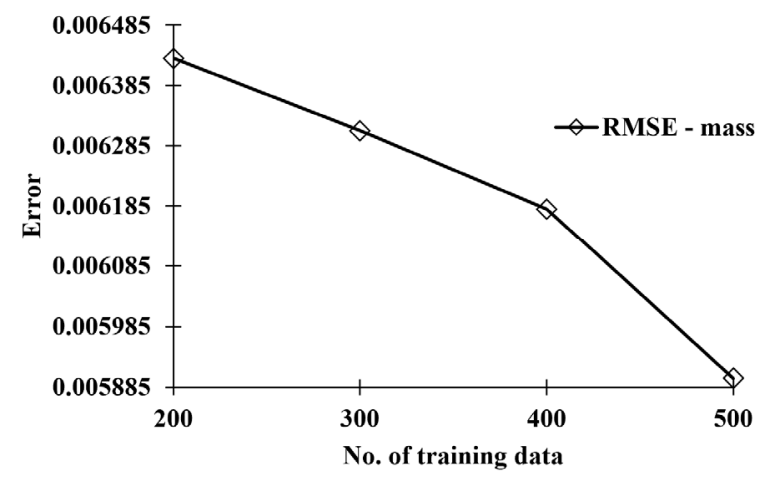

(a) RMSE on mass

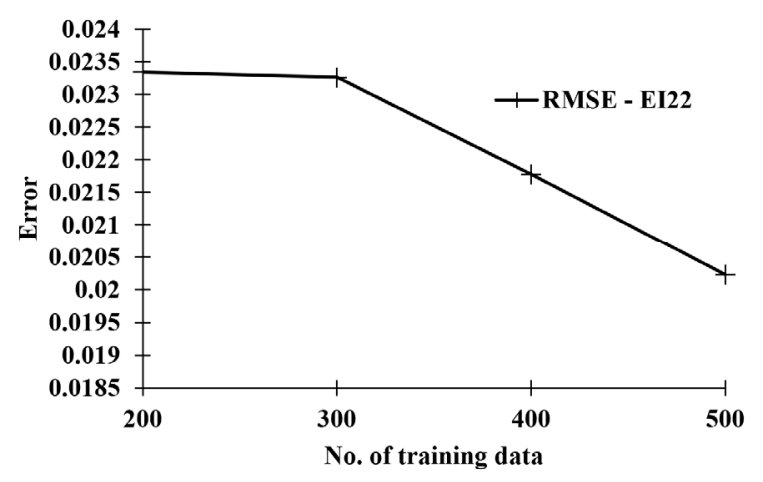

(c) RMSE on $\mathrm{EI}_{22}$

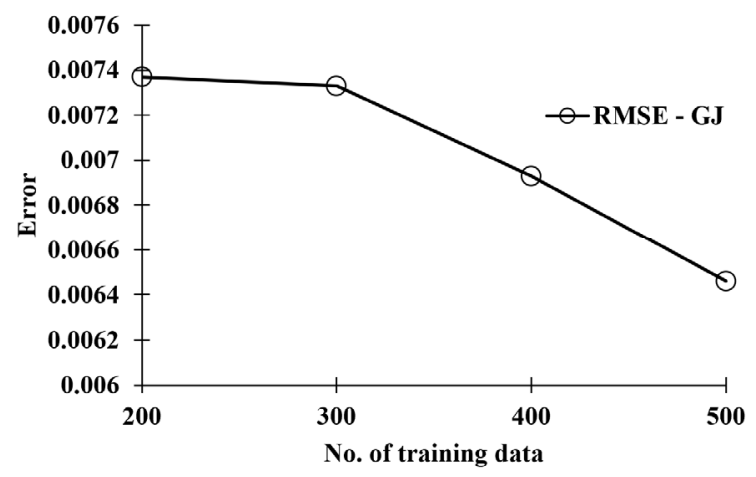

(b) RMSE on GJ

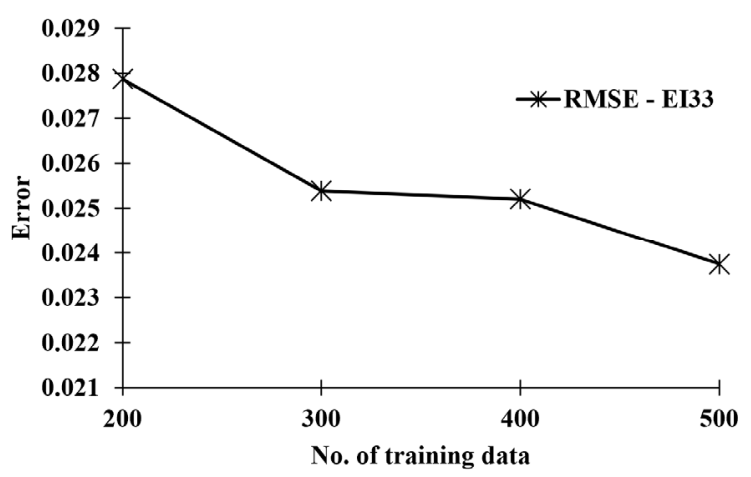

(d) RMSE on $\mathrm{EI}_{33}$

Figure 16. RMSE according to variation of training data.

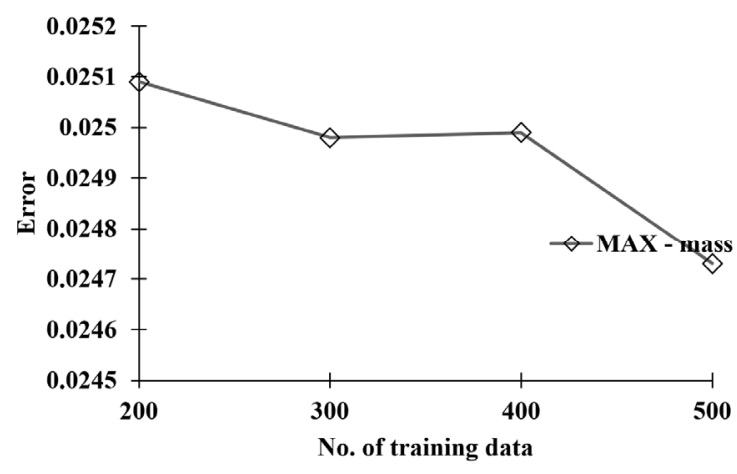

(a) MAX on mass

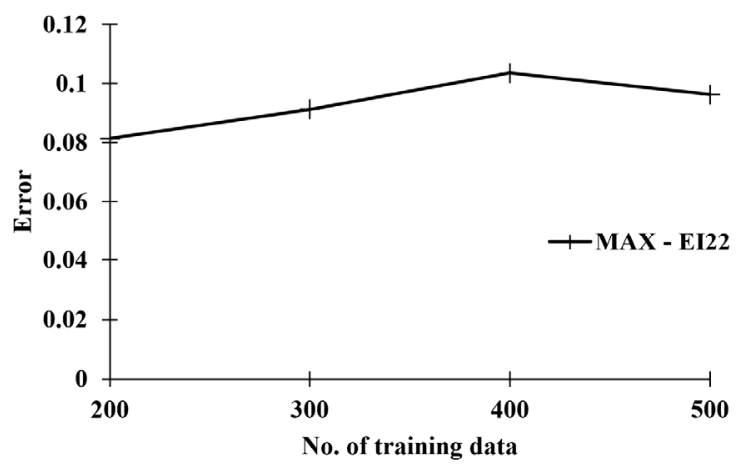

(c) $\mathrm{MAX}$ on $\mathrm{EI}_{22}$

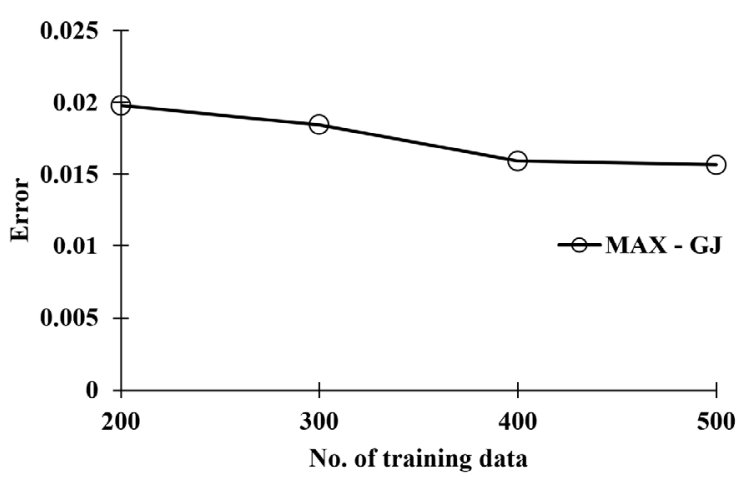

(b) MAX on GJ

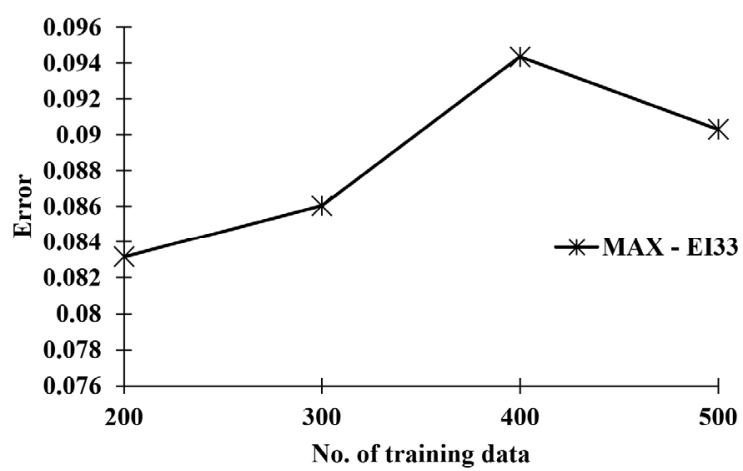

(d) $\mathrm{MAX}$ on $\mathrm{EI}_{33}$

Figure 17. Maximum error according to variation of training data. 
The results of Table 5 and Figure 16 represented that the RMSEs of Mass, GJ, EI 22 and $\mathrm{EI}_{33}$ were distributed in the range of 0.00590 to $0.00643,0.00646$ to $0.00737,0.02023$ to 0.02334 , and 0.02375 to 0.02787 , respectively, according to the variation of training data. It was found that the influence of the number of training data on the accuracy of the meta-model in the rotor blade design was higher than that of the SSP. RMSEs for all the design performance functions were enhanced as the number of training data was increased. The MAXs of Mass, GJ, $\mathrm{EI}_{22}$ and $\mathrm{EI}_{33}$ were distributed in the range of 0.02473 to 0.02509 , 0.01567 to $0.01977,0.08138$ to 0.09622 , and 0.08316 to 0.09031 , respectively, as represented in Table 4. Among the $\mathrm{MAX}$ results, however, $\mathrm{EI}_{22}$ and $\mathrm{EI}_{33}$ were deteriorated as the number of training data was increased, as shown in Figure 17. Such result was because some larger deviation data was included in the set of training data.

From Table 5 and Figure 17, it was found that the number of training data had larger influence on the RBFNN meta-model accuracy than the SSP in the approximation of rotor blade design problem. From the results of the rotor design approximation, it was able to conclude that the decrement of less than 100 times of number of design variables for the number of training data showed the inferior accuracy due to insufficient learning data for the RBFNN meta-model.

Among the learning parameters, the number of hidden layers were finally considered to evaluate the performance effects on the rotor blade design approximation. The error analyses were carried out applying one layer, two layers, and three layers to the RBFNN, respectively. In such those cases, 0.0 and 500 were applied in common for the SSP value and the number of training data. The results of RMSE and MAX were represented in Table 6 and Figures 18 and 19.

The results of Table 6 and Figure 18 represented that the RMSEs of Mass, GJ, EI 22 , and $\mathrm{EI}_{33}$ were distributed in the range of 0.00590 to $0.00591,0.00645$ to $0.00649,0.02023$ to 0.02025 , and 0.02375 to 0.02377 , respectively, according to the variation of hidden layer. The MAXs of Mass, GJ, $\mathrm{EI}_{22}$, and $\mathrm{EI}_{33}$ were distributed in the range of 0.02473 to 0.02491 , 0.01567 to $0.01597,0.09622$ to 0.09627 , and 0.09031 to 0.09041 , respectively, as represented in Table 6 and Figure 19. It was found that the influence of the number of hidden layers on the accuracy of the meta-model in the rotor blade design was scarcely detected comparing with the SSP and the number of training data. Although the effect of the number of hidden layers on the accuracy of the meta-model was insignificant, the RMSEs for all the design performance functions tended to slightly improve as the number of hidden layers was increased. The MAX results were also similar to the RSME results in case of the variation of the number of hidden layers.

Table 6. Results of accuracy according to variation of hidden layer.

\begin{tabular}{cccccc}
\hline Error Type & \# of Hidden Layers & Mass & GJ & EI $_{\mathbf{2 2}}$ & EI $_{\mathbf{3 3}}$ \\
\hline \multirow{3}{*}{ RMSE } & 1 & 0.00590 & 0.00649 & 0.02025 & 0.02377 \\
& 2 & 0.00591 & 0.00645 & 0.02022 & 0.02375 \\
& 3 & 0.00590 & 0.00646 & 0.02023 & 0.02375 \\
\hline \multirow{2}{*}{ MAX } & 1 & 0.02491 & 0.01597 & 0.09627 & 0.09041 \\
& 2 & 0.02477 & 0.01569 & 0.09621 & 0.09033 \\
& 3 & 0.02473 & 0.01567 & 0.09622 & 0.09031 \\
\hline
\end{tabular}




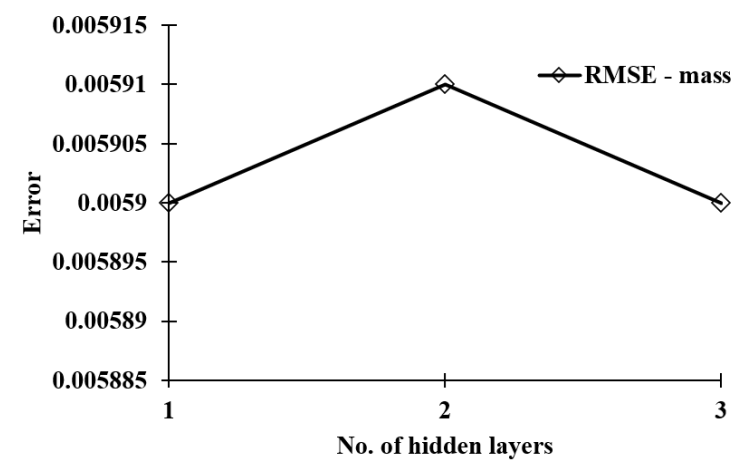

(a) RMSE on mass

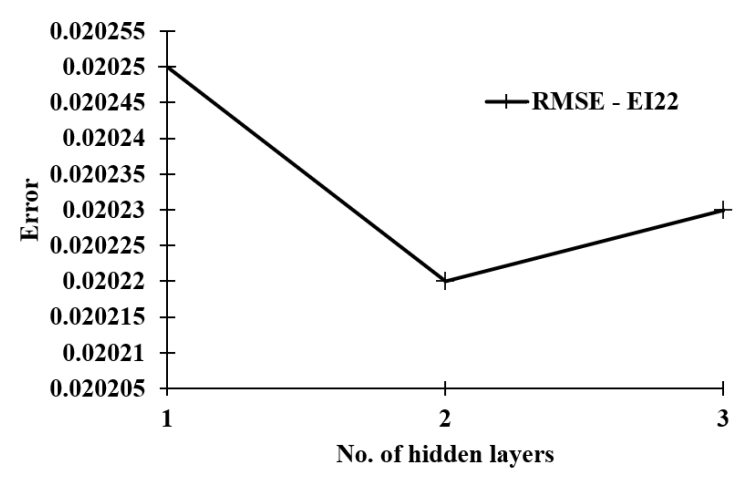

(c) RMSE on $\mathrm{EI}_{22}$

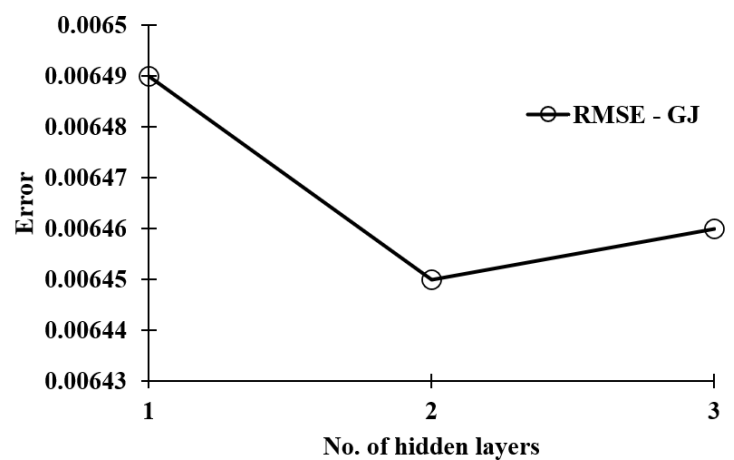

(b) RMSE on GJ

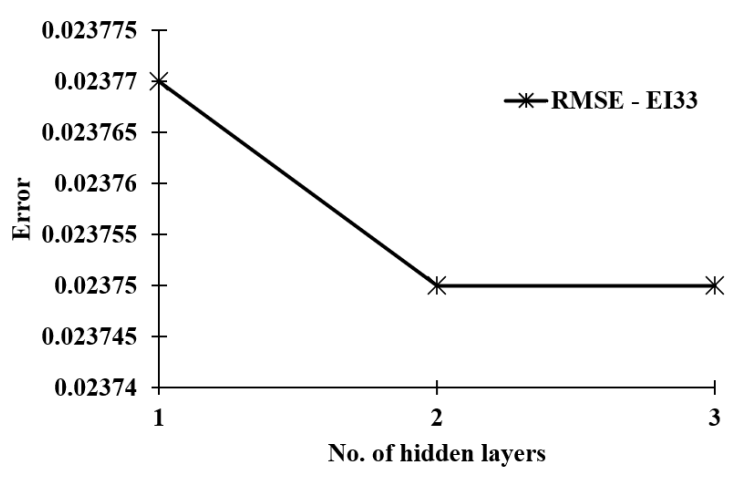

(d) RMSE on $\mathrm{EI}_{33}$

Figure 18. RMSE according to variation of hidden layer.

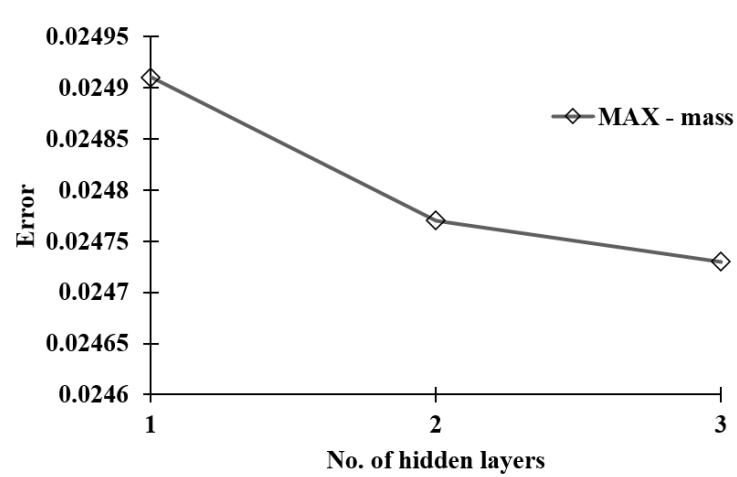

(a) MAX on mass

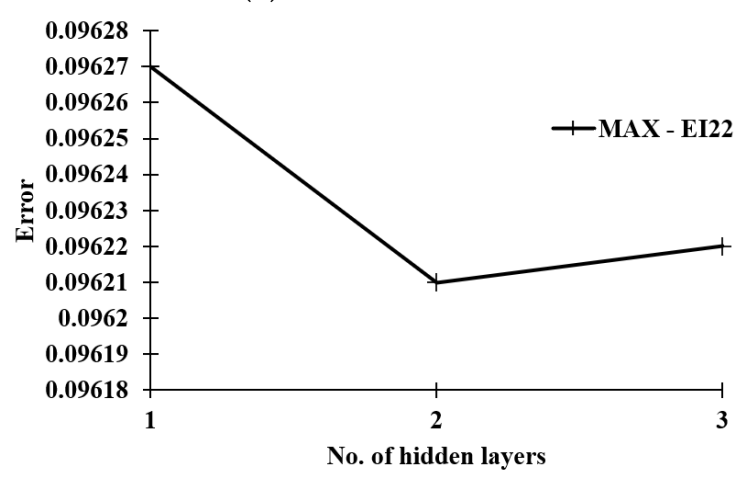

(c) MAX on $\mathrm{EI}_{22}$

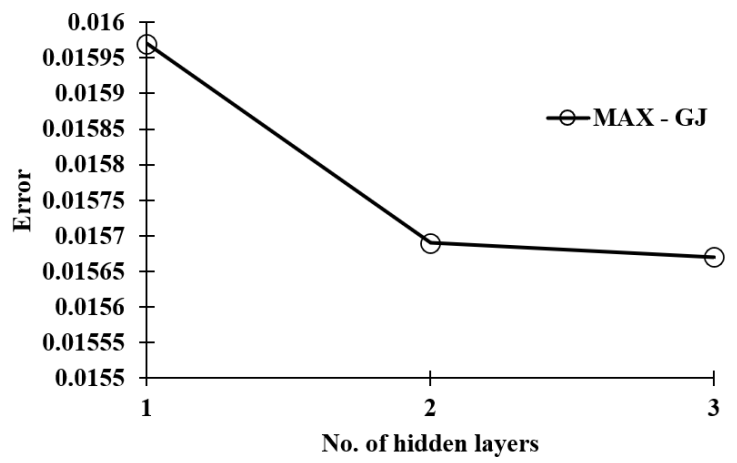

(b) MAX on GJ

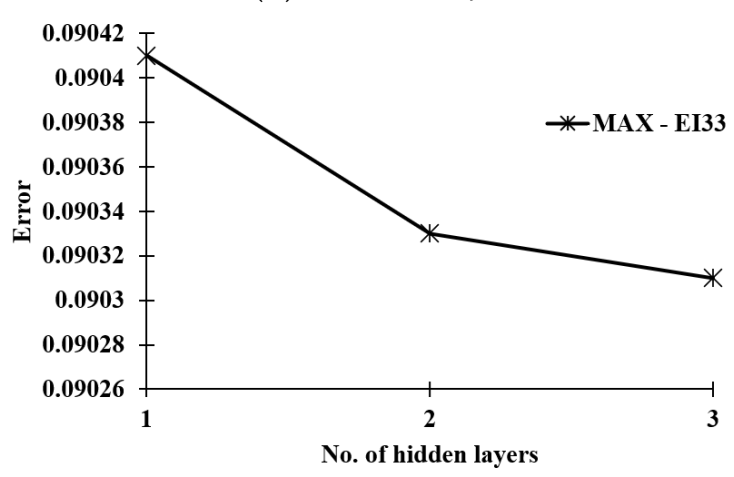

(d) $\mathrm{MAX}$ on $\mathrm{EI}_{33}$

Figure 19. Maximum error according to variation of hidden layer. 


\section{Closing Remarks}

This study identified the effect of various learning parameters such as the range of SSP, the number of training data, and the number of hidden layers on performances of RBFNN meta-model with respect to the design approximation. The rotor blade design problems were considered to review the characteristics of RBFNN approximation according to the variation of the learning parameters.

The RBFNN based meta-models were generated for the rotor blade design as the actual design problem. In the actual design problem of rotor blade with a number of performance functions, both the error analysis and the statistical analysis were performed. In the error analysis, two accuracy measures (RMSE and MAX) were used to evaluate the performance of RBFNN. With 500 training data and 90 testing data, RBFNN based meta-models of the rotor blade were fitted for each design performance function. From the statistical analysis results of the rotor design problem, it was found that the design performances of rotor blade were increased as the D-spar thickness was increased and the web location was decreased. In the results of the statistical analysis, it was also confirmed that the most sensitive design variable on both the mass and the flap-wise bending stiffness was the D-spar thickness while the most sensitive design variable on the torsional and chord-wise bending stiffnesses was the web location.

In regard of the learning parameters variation, first the SSP values were varied from zero to 0.1 with 0.025 step size, secondly the initial number of 500 training data was varied up to 200 training data with 100 data step size, and finally the RBFNN architecture was composed of one layer, two layers, and three layers respectively. From the results of the error analysis on the rotor design problem, it was found that the number of training data had larger influence on the RBFNN meta-model accuracy than the SSP variation while the number of hidden layers had little effect on the performances of RBFNN meta-model. Although the influence of SSP was not significant in the RBFNN approximation of the actual rotor blade design problem, it could be seen that the accuracy of the approximation was increased based on RMSE as the SSP was applied in the range of 0.075 to 0.1. From the results of the number of training data in the rotor blade design approximation, it was able to conclude that the decrement of less than 100 times of number of design variables for the number of training data showed the inferior accuracy due to insufficient learning data for the RBFNN meta-model. It was also found that RMSEs for all the design performance functions were enhanced as the number of training data was increased in the rotor blade design approximation. Regarding to the number of hidden layers, it was found that the influence of the number of hidden layers on the accuracy of the meta-model in the rotor blade design was scarcely detected comparing with the SSP and the number of training data. Although the effect of the number of hidden layers on the accuracy of the meta-model was insignificant, the RMSEs for all the design performance functions tended to slightly improve as the number of hidden layers was increased. This study results can help to suggest a reference index that can reasonably apply the learning parameter value in case of adopting the RBFNN to the design approximation.

Even though the RBFNN meta-model was utilized for the design optimizations and the reliability analyses, the role of learning parameters in the RBFNN meta-model was hardly investigated in the design approximation. In most previous studies, the values of parameters in RBFNN meta-model have been applied empirically or adjusted through simple estimation of the accuracy of RBFNN meta-model or comparison with the results of other meta-models. From this study results, the role of the important learning parameters such as the SSP, the number of training data, and the number of hidden layers was identified quantitatively using the rotor blade design problem. It was also clearly confirmed that the number of training data had larger influence on the RBFNN meta-model accuracy than the SSP variation while the number of hidden layers had little effect on the accuracy of RBFNN meta-model in the rotor blade design.

Author will carry out the application of RBFNN meta-model to some deep multimodal practical design and multidisciplinary design problems in further study. 
Funding: This research received no external funding.

Institutional Review Board Statement: Not applicable.

Informed Consent Statement: Not applicable.

Data Availability Statement: The data presented in this study are available in this article.

Acknowledgments: The results were supported the Ministry of Small and Medium-sized Enterprises (SMEs) and Startups (MSS), Korea, under the Collabo R\&D Program (No. S2910737) supervised by the Korea Technology and Information Promotion Agency (TIPA), and also supported by "Regional Innovation Strategy (RIS)" through the National Research Foundation of Korea (NRF) funded by the Ministry of Education (MOE).

Conflicts of Interest: The author declares no conflict of interest.

\section{References}

1. Broomhead, D.S.; Lowe, D. Radial Basis Functions, Multi-Variable Functional Interpolation and Adaptive Networks; Royal Signals and Radar Establishment: Malvern, UK, 1988.

2. Wan, C.; Harrington, P.B. Self-configuring radial basis function neural networks for chemical pattern recognition. J. Chem. Inform. Comput. Sci. 1999, 39, 1049-1056. [CrossRef]

3. Wu, Q.; Wang, X.; Chen, B.; Wu, H. Development of an RBFN-based neural-fuzzy adaptive control strategy for an upper limb rehabilitation exoskeleton. Mechatronics 2018, 53, 85-94. [CrossRef]

4. Shah, M.H.; Dang, X. Low-complexity deep learning and RBFN architectures for modulation classification of space-time block-code (STBC)-MIMO systems. Digit. Signal Process. 2020, 99, 102656. [CrossRef]

5. Sun, C.; Jin, Y.; Zeng, J.; Yu, Y. A two-layer surrogate-assisted particle swarm optimization algorithm. Soft comput. 2015, 19, 1461-1475. [CrossRef]

6. Kitayama, S.; Yamazaki, K. Sequential approximate robust design optimization using radial basis function network. Int. J. Mech. Mater. Des. 2014, 10, 313-328. [CrossRef]

7. Tang, Y.; Long, T.; Shi, R.; Wu, Y.; Wang, G.G. Sequential radial basis function-based optimization method using virtual sample generation. J. Mech. Des. 2020, 142, 1-13. [CrossRef]

8. Hardy, R.L. Multiquadratic equations of topography and other irregular surfaces. J. Geophys. Res. 1971, 76, 1905-1915. [CrossRef]

9. Fang, H.; Horstemeyer, M.F. Global response approximation with radial basis functions. Eng. Optim. 2006, 38, 407-424. [CrossRef]

10. Dyn, N.; Levin, D.; Rippa, S. Numerical procedures for surface fitting of scattered data by radial basis functions. SIAM J. Sci. Comput. 1986, 7, 639-659. [CrossRef]

11. Gutmann, H.M. A radial basis function method for global optimization. J. Glob. Optim. 2001, 19, 201-227. [CrossRef]

12. Hussain, M.F.; Barton, R.R.; Joshi, S.B. Metamodeling: Radial basis functions versus polynomials. Eur. J. Oper. Res. 2002, 138, 142-154. [CrossRef]

13. Jin, R.; Chen, W.; Simpson, T.W. Comparative studies of metamodelling techniques under multiple modeling criteria. Struct. Multidiscipl. Optim. 2001, 23, 1-13. [CrossRef]

14. Mullur, A.A.; Messac, A. Extended radial basis functions: More flexible and effective metamodeling. AIAA J. 2005, 43, 1306-1315. [CrossRef]

15. Fasshauer, G.E.; Zhang, J.G. On choosing optimal shape parameters for RBF approximation. Numer. Algorithms 2007, 45, 345-368. [CrossRef]

16. Lee, D.J.; Song, C.Y.; Lee, K. Surrogate model based approximate optimization of passive type deck support frame for offshore plant float-over installation. J. Ocean Eng. Technol. 2021, 35, 1-10. [CrossRef]

17. Song, C.Y. Reliability analysis for structure design of automatic ocean salt collector using sampling method of Monte Carlo simulation. J. Ocean Eng. Technol. 2020, 34, 316-324. [CrossRef]

18. Song, C.Y.; Lee, D.J.; Lee, J.S.; Kim, E.M.; Choi, B.Y. Evaluation of structural design enhancement and sensitivity of automatic ocean salt collector according to design of experiments. J. Ocean Eng. Technol. 2020, 34, 253-262. [CrossRef]

19. Mirjalili, S.; Lewis, A.; Dong, J.S. Confidence based robust optimization using multi objective meta-heuristics. Swarm Evol. Comput. 2018, 43, 109-126. [CrossRef]

20. Song, C.Y.; Lee, J. Reliability-based design optimization of knuckle component using conservative method of moving least squares meta-models. Probabilistic Eng. Mech. 2011, 26, 364-379. [CrossRef]

21. Fornberg, B.; Zuev, J. The Runge phenomenon and spatially variable shape parameters in RBF interpolation. Comput. Math. Appl. 2007, 54, 379-398. [CrossRef]

22. Vidya, L.; Vivekanand, V.; Shyamkumar, U.; Deepak, M. RBF-network based sparse signal recovery algorithm for compressed sensing reconstruction. Neural Netw. 2015, 63, 66-78. [CrossRef]

23. Rouhani, M.; Sappa, A.D.; Boyer, E. Implicit B-spline surface reconstruction. IEEE Trans. Image Process. 2014, 24, 22-32. [CrossRef] [PubMed] 
24. Belardo, M.; Beretta, J.; Marano, A.D.; Diodati, G.; Paletta, N.; Di Palma, L. On the preliminary structural design strategy of the wing of the Next-Generation Civil Tiltrotor technology demonstrator. Int. J. Aeronaut. Space Sci. 2020, 22, 613-624. [CrossRef]

25. Belardo, M.; Marano, A.D.; Beretta, J.; Diodati, G.; Graziano, M.; Capasso, M.; Ariola, P.; Orlando, S.; Di Caprio, F.; Paletta, N.; et al. Wing structure of the Next-Generation Civil Tiltrotor: From concept to preliminary design. Aerospace 2021, 8, 102. [CrossRef]

26. Müller, B.; Reinhardt, J. Neural Networks; Springer: Berlin, Germany, 1990.

27. Smith, M. Neural Networks for Statistical Modeling; Thomson: London, UK, 1996.

28. Kumar, S. Neural Networks; McGraw-Hill: New York, NY, USA, 2010.

29. Mullur, A.A.; Messac, A. Metamodeling using extended radial basis functions: A comparative approach. Eng. Comput. 2006, 21, 203-217. [CrossRef]

30. Lee, J.; Shin, K.H. A conservative method of wavelet neural network based meta-modeling in constrained approximate optimization. Comput. Struct. 2011, 89, 109-126. [CrossRef]

31. Ackley, D.H. A Connectionist Machine for Genetic Hillclimbing; Kluwer Academic Publishers: Boston, MA, USA, 1987.

32. Lee, J.; Jeong, H.; Choi, D.-H.; Volovoi, V.; Mavris, D. An enhancement of constraint feasibility in BPN based approximate optimization. Comput. Methods Appl. Mech. Eng. 2007, 196, 2147-2160. [CrossRef]

33. Cesnik, C.E.S.; Hodges, D.H. VABS: A new concept for composite rotor blade cross-sectional modeling. J. Am. Helicop. Soc. 1997, 42, 27-38. [CrossRef] 